\title{
Lightning Charge Retrievals: Dimensional Reduction, LDAR Constraints, and a First Comparison with LIS Satellite Data
}

\author{
W. J. Koshak ${ }^{1}$, E. P. Krider ${ }^{2}$, N. Murray ${ }^{2}$, and D. J. Boccippio ${ }^{1}$ \\ 'NASA Marshall Space Flight Center, Huntsville, Alabama \\ ${ }^{2}$ University of Arizona, Tucson, Arizona
}

February 2, 2007

Corresponding author address: Dr. William Koshak, Earth Science Office, VP61, NASA Marshall Space Flight Center, Robert Cramer Research Hall, 320 Sparkman Drive, Huntsville, AL 35805. email address: william.koshak@nasa.gov 
Abstract. A "dimensional reduction" (DR) method is introduced for analyzing lightning field changes $(\Delta E s)$ whereby the number of unknowns in a discrete two-charge model is reduced from the standard eight $\left(x, y, z, Q, x^{\prime}, y^{\prime}, z^{\prime}, Q^{\prime}\right)$ to just four $(x, y, z, Q)$. The four unknowns $(x, y, z, Q)$ are found by performing a numerical minimization of a chi-square function. At each step of the minimization, an Overdetermined Fixed Matrix (OFM) method is used to immediately retrieve the best "residual source" $\left(x^{\prime}, y^{\prime}, z^{\prime}, Q^{\prime}\right)$, given the values of $(x, y, z, Q)$. In this way, all 8 parameters $\left(x, y, z, Q, x^{\prime}, y^{\prime}, z^{\prime}, Q^{\prime}\right)$ are found, yet a numerical search of only 4 parameters $(x, y, z, Q)$ is required. The DR method has been used to analyze lightning-caused $\triangle E s$ derived from multiple ground-based electric field measurements at the NASA Kennedy Space Center (KSC) and USAF Eastern Range (ER). The accuracy of the DR method has been assessed by comparing retrievals with data provided by the Lightning Detection And Ranging (LDAR) system at the KSC-ER, and from least squares error estimation theory, and the method is shown to be a useful "stand-alone" charge retrieval tool. Since more than one charge distribution describes a finite set of $\Delta E S$ (i.e., solutions are non-unique), and since there can exist appreciable differences in the physical characteristics of these solutions, not all DRsolutions are physically acceptable. Hence, an alternative and more accurate method of analysis is introduced that uses LDAR data to constrain the geometry of the charge solutions, thereby removing physically unacceptable retrievals. The charge solutions derived from this method are shown to compare well with independent satellite- and ground-based observations of lightning in several Florida storms. 


\section{Introduction}

Lightning-caused changes in the surface electric field have been used to determine the characteristics (magnitude, polarity, location) of the charges that are effectively deposited by lightning [e.g., Wilson, 1916, 1920; Workman and Holzer, 1942; Jacobson and Krider, 1976; Krehbiel et al., 1979; Krehbiel, 1981, 1986; Maier and Krider, 1986; Koshak and Krider, 1989, 1994; Murphy et al., 1996; Koshak et al., 1999]. Conventional charge retrieval algorithms minimize a nonlinear, chi-square function to find the optimum parameters, e.g. charge locations and values, of an assumed model of the source. Retrieval accuracy depends in part on the model and on the error in the lightning-caused electric field changes $(\Delta E s)$. For the ground-based network of electric field sensors (field mills) at the NASA Kennedy Space Center (KSC) and the USAF Eastern Range (ER), and for the algorithm used to compute the $\Delta E s$ from the electric field records (e.g., see Koshak and Krider, 1989), the estimated error in $\Delta E$ is typically under $15 \%$. Normally, a simple charge model is used to fit the $\Delta E S$ to within this error, and if this approach is successful, it complies with a principle known as Occam's Razor. The $14^{\text {th }}$ century English philosopher and Franciscan monk William of Occam, has stated "Pluralitas non est ponenda sine neccesitate" or "plurality should not be posited without necessity." From this point of view, a simple model composed of just one or two spherically symmetric sources would be better than a more complicated solution if the simple solution described the observed $\Delta E S$ to within $15 \%$. In effect, Occam's Razor uses only the simplest models to describe the observations and ignores all other possibilities.

In this paper, section 2 briefly reviews the goodness-of-fit method that is customarily used to determine the optimum parameters of a charge model, and includes a description of the one-charge model (also called the point-charge or "Q-model"), and the two-charge model. 
Section 3a reviews a 4-station analytic method that can be used to find the parameters of the onecharge model (Krehbiel et al,, 1979), section 3b generalizes this method to 4 or more stations, and section $3 \mathrm{c}$ describes an Overdetermined Fixed Matrix (OFM) approach that is most desirable. Section 4 discusses tests of the OFM method using simulated sources with and without random errors in the $\Delta E s$. Section 5 introduces a "Dimensional Reduction" (DR) method that uses the OFM approach to effectively reduce the number of unknowns in a general eight parameter (two-charge) model to just four unknown parameters. Section 6 uses the DR method to analyze over one hundred lightning flashes in a thunderstorm at the KSC-ER and then discusses the advantages and limitations of the DR method.

As an alternative to the DR method, section 7 introduces a simple charge retrieval algorithm that uses KSC-ER Lightning Detection And Ranging (LDAR) data to constrain the charge locations, and a linear least-squares matrix inversion to determine the charge values. Section 8 applies this (computationally fast) method to derive charge solutions for a variety of Florida thunderstorms, and compares the results to independent satellite- and ground-based lightning observations. Finally, section 9 summarizes this work.

\section{Goodness-of-Fit Analyses}

A method for retrieving the optimum parameters of an assumed lightning charge model by numerically minimizing a nonlinear, chi-square error function was introduced by Jacobson and Krider (1976). Given $m$ field change observations at the ground (i.e., $\Delta E_{i}, i=1, \ldots, m$ ) the reduced chi-square function is

$$
\chi^{2}(\mathbf{a})=\frac{1}{m-n} \sum_{i=1}^{m} \frac{\left(M_{i}(\mathbf{a})-\Delta E_{i}\right)^{2}}{\sigma_{i}^{2}}
$$


where $M_{i}$ (a) is the model field change at the $i^{\text {th }}$ site, and $\sigma_{i}$ is the observation error in $\Delta E_{i}$. The model $M_{i}$ is a function of $n$ unknown model parameters that are contained in the $n$-vector $\mathbf{a}=\left(a_{1}, \ldots, a_{n}\right)$. The difference $(m-n)$ is termed the "degrees of freedom."

Simple examples of the model $M_{i}(\mathbf{a})$, and the ones of greatest interest here, are the oneand two-charge models. Denoting the one-charge model by the subscript "1", it can be written

$$
M_{1 i}\left(\mathbf{a}_{1}\right)=\frac{-Q z}{2 \pi \varepsilon_{o}\left[\left(X_{i}-x\right)^{2}+\left(Y_{i}-y\right)^{2}+z^{2}\right]^{3 / 2}},
$$

where $\left(X_{i}, Y_{i}\right)$ is the location of the observation $\Delta E_{i}$, and $\mathbf{a}_{1}=(x, y, z, Q)$. This model assumes that the ground is a flat conducting plane of infinite extent. We use the standard physics convention so that $M_{1 i}$ is the change in the vertical electric field at the $i^{\text {th }}$ site; hence, a monopolar cloud-to-ground lightning that transfers negative charge to ground would effectively deposit a single positive charge $(Q>0)$ at location $(x, y, z)$ above the conducting plane, and thereby results in a negative change in field, $M_{1 i}<0$. Using superposition and (2), the 8parameter two-charge model can be written as

$$
M_{2 i}\left(\mathbf{a}_{2}\right)=\frac{-1}{2 \pi \varepsilon_{o}}\left[\frac{Q z}{\left[\left(X_{i}-x\right)^{2}+\left(Y_{i}-y\right)^{2}+z^{2}\right]^{3 / 2}}+\frac{Q^{\prime} z^{\prime}}{\left[\left(X_{i}-x^{\prime}\right)^{2}+\left(Y_{i}-y^{\prime}\right)^{2}+z^{\prime 2}\right]^{3 / 2}}\right],
$$

where $\mathbf{a}_{2}=\left(x, y, z, Q, x^{\prime}, y^{\prime}, z^{\prime}, Q^{\prime}\right)$.

According to the statistical theory discussed in Bevington and Robinson (2003, Chapter 11), a value of $\chi^{2} \cong 1$ implies that the model-fitting function $M_{i}(\mathbf{a})$ in (1) is a good description of the observations provided that the values of $\sigma_{i}$ are accurately estimated. 
To understand how well one must model a given set of $\Delta E s$ so that $\chi^{2} \cong 1$, suppose that the error in $\Delta E$ is constant, that is, $\sigma_{i}=\sigma ; i=1, \ldots, m$. In this case (1) becomes

$$
\chi^{2}(\mathbf{a})=\frac{1}{(m-n) \sigma^{2}} \sum_{i=1}^{m}\left(M_{i}(\mathbf{a})-\Delta E_{i}\right)^{2}
$$

and a "good fit" (i.e., $\chi^{2} \cong 1$ ) implies that

$$
\sum_{i=1}^{m}\left(M_{i}(\mathbf{a})-\Delta E_{i}\right)^{2} \cong(m-n) \sigma^{2}
$$

In addition, note that the rms modeling error, $R$ (in $\mathrm{V} / \mathrm{m}$ ), is

$$
R \equiv\left[\frac{1}{m} \sum_{i=1}^{m}\left(M_{i}(\mathbf{a})-\Delta E_{i}\right)^{2}\right]^{1 / 2}
$$

Substituting the right hand side of (5) into (6) gives the approximate value of $R$ required to obtain a "good fit," that is,

$$
R \cong \xi \sigma \Rightarrow \text { "good fit" ; } 1 \leq n \leq m
$$

where $\xi \equiv(1-n / m)^{1 / 2}$. For the $m=31$ sensors in the field measuring network at the KSC-ER, $\xi$ takes on the values shown in Table 1. Note that $R$ must always be less than $\sigma$ to be viewed as a good fit; i.e., $0 \leq \xi<1$ always holds. In addition, note that $\xi$ decreases as $n$ increases. This means that a complicated model must describe the $\Delta E S$ more accurately than a simple model in order to be regarded as a "good fit." For example, the one-charge model $(n=4)$ has $\xi=0.933$, but the two-charge model $(n=8)$ has $\xi=0.861$. The improvement in modeling accuracy that is 
required to maintain a "good fit" is not linear in $n$. As $n$ increases, $\xi$ decreases at a progressively faster rate because the derivative $d \xi / d n$ is inversely proportional to $\xi$, i.e.

$$
\frac{d \xi}{d n}=-\frac{1}{2 m}\left(1-\frac{n}{m}\right)^{-\frac{1}{2}}=-\frac{1}{2 m \xi} ; \quad 1 \leq n \leq m
$$

In summary, when applying models of increasing complexity, one must be sure that they can reduce the value of $R$ by an amount that is sufficient to obtain a good fit. In the limit as $n \rightarrow m$, the model must describe the $\Delta E s$ perfectly in order to achieve a good fit. Again, rather than increasing the model complexity, $n$, Occam's Razor suggests one should reduce model complexity as much as possible while still trying to maintain a good fit.

\section{Analytic Solution to the Point Charge Model}

a. Four station solution

Appendix 1 of Krehbiel et al. (1979) describes an analytic method for determining the four unknown parameters of the Q-model from four ground-based observations of $\Delta E$. This approach generalized an earlier method due to Fitzgerald (1957) that had certain limitations. By replacing the model field changes $M_{1 i}$ in (2) with the (absolute value of) the real field changes $\Delta E_{i}$ produced by a monopolar flash, one obtains

$$
\left|\Delta E_{i}\right|=\frac{|Q| z}{2 \pi \varepsilon_{o}\left[\left(X_{i}-x\right)^{2}+\left(Y_{i}-y\right)^{2}+z^{2}\right]^{3 / 2}} .
$$

Rearranging terms, and then differencing pairs of the $i=1, \ldots, 4$ equations, Krehbiel et al. (1979) obtained 


$$
2\left(X_{i}-X_{j}\right) x+2\left(Y_{i}-Y_{j}\right) y+U_{i j} \eta=X_{i}^{2}+Y_{i}^{2}-X_{j}^{2}-Y_{j}^{2},
$$

where $U_{i j}=\left[\left(4 \pi \varepsilon_{o}\right)^{-2 / 3}\left(\left|\Delta E_{i}\right|^{-2 / 3}-\left|\Delta E_{j}\right|^{-2 / 3}\right)\right.$, and $\eta=(2|Q| z)^{2 / 3}$. [Note: Krehbiel et al. actually omit all the absolute value signs shown here, thereby restricting their result to the case $Q>0$. The results shown here are valid for any polarity of $Q$. ] Taking a combination of 4 sensors 2 at a time gives $4 ! /(2 ! 2 !)$ or 6 possible combinations. Krehbiel et al. pick 3 of these 6 to obtain 3 equations in 3 unknowns, i.e.

$$
\left[\begin{array}{ccc}
2\left(X_{1}-X_{2}\right) & 2\left(Y_{1}-Y_{2}\right) & U_{12} \\
2\left(X_{1}-X_{3}\right) & 2\left(Y_{1}-Y_{3}\right. & U_{13} \\
2\left(X_{1}-X_{4}\right) & 2\left(Y_{1}-Y_{4}\right) & U_{14}
\end{array}\right]\left[\begin{array}{l}
x \\
y \\
\eta
\end{array}\right]=\left[\begin{array}{c}
X_{1}^{2}+Y_{1}^{2}-X_{2}^{2}-Y_{2}^{2} \\
X_{1}^{2}+Y_{1}^{2}-X_{3}^{2}-Y_{3}^{2} \\
X_{1}^{2}+Y_{1}^{2}-X_{4}^{2}-Y_{4}^{2}
\end{array}\right],
$$

or in matrix-vector notation

$$
\mathbf{A v}=\mathbf{b}
$$

The column vector solution for the unknowns is $\mathbf{v}=\operatorname{col}(x, y, \eta)=\mathbf{A}^{-1} \mathbf{b}$. Substituting the $(x, y)$ derived from this solution into (9) and noting that $|Q| z=\frac{1}{2} \eta^{3 / 2}$, one can determine $z$. With $z$ and the definition of $\eta$ one obtains $|Q|$. The sign of $Q$ is the same as the sign of the $\triangle E s$, hence, the problem is solved. Note that this method can only be used when the $\Delta E s$ have the same polarity, i.e., the solution is only possible for a monopole source.

\section{b. Overdetermined Variable Matrix (OVM) approach}

As discussed above, Krehbiel et al. (1979) used four ground-based $\Delta E$ observations to obtain the four unknown parameters $(x, y, z, Q)$ in the Q-model. However, retrieval accuracy can be improved if all $m$ observations are used at the same time. This assumes that the observation 
errors are all similar in magnitude, and that the distances between the observation sites are sufficiently large so that the $\mathbf{A}$ matrix is not ill-conditioned. Inversion of an ill-conditioned matrix produces large retrieval errors because small eigenvalues magnify observation errors (Twomey, 1977; Chapter 6).

Instead of extending their analytic solution to analyze all $m$ observations of $\Delta E$ at the same time, Krehbiel et al. (1979) computed several four-station solutions using different combinations of sensors, and then averaged the results. This approach is unconventional in inversion theory (Twomey, 1977), and it is also computationally intensive. For $m=31$ sensors (the number of field mills in the network at the KSC-ER), there are $m ! /[(m-4) ! 4 !]=31,465$ four-station solutions to compute. It is preferable to extend the difference scheme in (11) so that all $m$ observations can be analyzed at the same time; this gives the overdetermined system of equations

$$
\left[\begin{array}{ccc}
2\left(X_{1}-X_{2}\right) & 2\left(Y_{1}-Y_{2}\right) & U_{12} \\
\cdot & \cdot & \cdot \\
\cdot & \cdot & \cdot \\
2\left(X_{1}-X_{m}\right) & 2\left(Y_{1}-Y_{m}\right) & U_{1 m}
\end{array}\right]\left[\begin{array}{l}
x \\
y \\
\eta
\end{array}\right]=\left[\begin{array}{c}
X_{1}^{2}+Y_{1}^{2}-X_{2}^{2}-Y_{2}^{2} \\
\cdot \\
\cdot \\
X_{1}^{2}+Y_{1}^{2}-X_{m}^{2}-Y_{m}^{2}
\end{array}\right] .
$$

Defining again the matrix on the left hand side as $\mathbf{A}$, and the vector on the right hand side as $\mathbf{b}$, one can pre-multiply (13) by $\widetilde{\mathbf{A}}$ (the transpose of $\mathbf{A}$ ) and invert to obtain the solution

$$
\mathbf{v}=(\widetilde{\mathbf{A}} \mathbf{A})^{-1} \widetilde{\mathbf{A}} \mathbf{b}
$$

This is a least-squares solution (Twomey, 1977; Chapter 6), and the optimum values of $(x, y, z, Q)$ can be computed from $\mathbf{v}$ as discussed in section 3a. Again, since the solution (14) uses all $m$ observations to obtain the four model parameters, it is expected to provide a more realistic answer than any single solution derived from (12). Furthermore, external constraints can 
be added to the solution process if desired (see Twomey [1977] for an introduction to constrained linear inversions).

\section{c. Overdetermined Fixed Matrix (OFM) approach}

The difference equations (10) were formed in order to remove the nonlinear terms $x^{2}, y^{2}$, and $z^{2}$; however, these differences are not required to solve the problem analytically. An alternate and more practical solution (that we will ultimately use in section 5 to follow) can be found. We begin with (9), i.e., we assume that the source producing the $\Delta E s$ is a monopole so that all the field changes have the same sign. Raising (9) to the $-2 / 3$ power gives

$$
\begin{aligned}
d_{i} & =w\left[\left(X_{i}-x\right)^{2}+\left(Y_{i}-y\right)^{2}+z^{2}\right] \\
& =\left(X_{i}^{2}+Y_{i}^{2}\right) w-2 X_{i} w x-2 Y_{i} w y+w r^{2},
\end{aligned}
$$

where

$$
d_{i} \equiv\left|\Delta E_{i}\right|^{-2 / 3}, \quad w \equiv\left(\frac{2 \pi \varepsilon_{o}}{|Q| z}\right)^{2 / 3}, r^{2} \equiv x^{2}+y^{2}+z^{2}
$$

For $i=1, \ldots, m$ sensors, $(15)$ yields the $(m \times 4)$ system

$$
\left[\begin{array}{c}
d_{1} \\
\cdot \\
\cdot \\
d_{m}
\end{array}\right]=\left[\begin{array}{cccc}
\left(X_{1}^{2}+Y_{1}^{2}\right) & -2 X_{1} & -2 Y_{1} & 1 \\
\cdot & \cdot & \cdot & \cdot \\
\cdot & \cdot & \cdot & \cdot \\
\left(X_{m}^{2}+Y_{m}^{2}\right) & -2 X_{m} & -2 Y_{m} & 1
\end{array}\right]\left[\begin{array}{c}
w \\
w x \\
w y \\
w r^{2}
\end{array}\right],
$$

that can be written in matrix-vector form as

$$
\mathbf{d}=\mathbf{T s} \text {. }
$$


Premultiplying (18) by $\widetilde{\mathbf{T}}$ and inverting gives

$$
\mathbf{s}=\mathbf{N d}
$$

where $\mathbf{N} \equiv(\widetilde{\mathbf{T} T})^{-1} \widetilde{\mathbf{T}}$ is termed the "network matrix" because its elements are determined only by the geometry of the sensor network. The model parameters can be obtained from the elements of $\mathbf{s}=\left(s_{1}, s_{2}, s_{3}, s_{4}\right)$ as follows

$$
\begin{aligned}
& x=s_{2} / s_{1} \\
& y=s_{3} / s_{1} \\
& z=\left(s_{1} s_{4}-s_{2}^{2}-s_{3}^{2}\right)^{1 / 2} / s_{1} \\
& Q=\left(2 \pi \varepsilon_{o} \Delta E_{1}\right) /\left[\left(s_{1}^{2} s_{4}-s_{1} s_{2}^{2}-s_{1} s_{3}^{2}\right)^{1 / 2}\left|\Delta E_{1}\right|\right] .
\end{aligned}
$$

Note that the sign of $Q$ is the same as any of the $\Delta E s$, for example $\Delta E_{1} /\left|\Delta E_{1}\right|$ as shown in the last equation of (20).

A distinct advantage of the solution in (19) over that provided in (14) is that the network matrix $\mathbf{N}$ need only be computed once (for a given network geometry), whereas the matrix $\mathbf{A}$ must be computed for each lightning flash because it contains the $\Delta E$ observations. Since the retrieval accuracy of a matrix inversion depends on the mathematical condition of the matrix, it is clearly better to have this condition fixed (and well understood), as in the case of $\mathbf{N}$, rather than have it vary from flash to flash as with $\mathbf{A}$. Of course, if any sensor fails or is removed from the analysis for any other reason, then $\mathbf{N}$ will change.

In summary, (20) provides a way to use all $m \Delta E$ observations to infer the optimum source parameters $(x, y, z, Q)$. Whereas the method discussed in section $3 \mathrm{~b}$ obtains a solution by removing the nonlinear terms $\left(x^{2}, y^{2}\right.$, and $\left.z^{2}\right)$ through differencing, and results in a variable $\mathbf{A}$ matrix from flash to flash, the OFM method provided in this section demonstrates that a solution 
can be obtained without having to remove the nonlinear terms. The OFM approach eliminates the variable A matrix altogether, and effectively replaces it with the "fixed" network matrix $\mathbf{N}$. This matrix only has to be computed once for any given network geometry, and it is not difficult or time consuming to compute because it involves the inversion of one small $(4 \times 4)$ matrix $\widetilde{\mathbf{T} T}$. After $\mathbf{N}$ is computed for a particular network, no other matrix inversions are required to retrieve monopolar flashes detected by that network. All the $\Delta E$ values are contained in the $\mathbf{d}$ vector and each monopolar solution is obtained from the product $\mathbf{N d}=\mathbf{s}$, followed by application of $(20)$.

\section{Simulated Tests of the OFM Approach}

Before using the OFM method to help analyze natural lightning, we tested it on a number of simulated monopole sources that were placed over the sensor network at the KSC-ER. With no errors in the simulated $\Delta E$ observations, the OFM method retrieved the parameters of an arbitrary monopole exactly (apart from very small round-off errors). When a $5 \%$ random error was added to each of the simulated $\Delta E$ observations, the $\Delta E$ modeling errors and solution retrieval errors shown in Figure 1 were obtained.

To cover the region in Figure 1, we placed sources on a horizontal grid that had dimensions $59 \mathrm{~km} \times 59 \mathrm{~km}$ (resolution $1 \mathrm{~km}$ ) and an altitude of $6.5 \mathrm{~km}$. The error statistics were generated by analyzing 100 monopole sources (each $25 \mathrm{C}$ ) at each grid point. To obtain a set of simulated $\Delta E$ observations from a source, we computed the field at each site due to the source, and then added a random error to each field value. The random errors were chosen from a normal distribution that had a mean of zero, and a standard deviation equal to $5 \%$ of the field value. The

percent $\Delta E$ modeling error, $P$, given in Figure 1a is defined as $P \equiv 100 \times|\Delta \mathbf{E}-\mathbf{M}| /|\Delta \mathbf{E}|$, where 
$\Delta \mathbf{E}$ and $\mathbf{M}$ are $m$-vectors of the simulated and model field changes, respectively. Using the key in Figure 2, note that horizontal and vertical errors in the retrieved distances are only 0.1 to 0.5 $\mathrm{km}$ over the network, and the errors in $Q$ are less than $1 \mathrm{C}$ over a substantial portion of the grid. For the most part, the errors in horizontal distance, altitude, and $Q$ increase outside the network. This behavior is typical for all inversion algorithms (see, for example, the tests of the multipole method in Appendix A of Koshak et al., 1999). In addition, note that it is more appropriate to plot $P$ (Figure 1a) rather than the reduced chi-square. This is because the retrieval errors are directly calculated in these simulations, so the chi-square statistical theory for rating solution quality and estimating retrieval errors is not needed. Nonetheless, $P$ and $\chi^{2}$ are mathematically dependant variables. For an assumed fixed $\sigma$ equal to $5 \%$ of the rms $\Delta E$ value for a monopolar flash, the green regions in Figure 1a $(P \sim 5 \%$ to $10 \%)$ correspond to a range in $\chi^{2}$ of 1.15 to 4.59 which typically indicates physically reasonable fits.

Because the OFM approach is essentially a single matrix inversion method for retrieving monopoles, it (reasonably) requires that all $\Delta E s$ be nonzero and of the same polarity. In addition, errors in $\Delta E$ can cause the difference $\left(s_{1} s_{4}-\left[s_{2}^{2}+s_{3}^{2}\right]\right)$ in (20) to be negative, and this produces a complex number for the altitude, $z$. Errors in $\Delta E$ can also make the difference too small or too large, thereby producing a value of $z$ that is erroneous based on physical grounds. Hence, if any of the above conditions (one or more zero $\Delta E s$, bipolar $\Delta E s$, a complex or unphysical $z$ ) occur, that test source has been ignored and another source has been generated as a replacement. To generate the statistics in Figure 1, we had to replace 96,179 sources. That is, we analyzed a total of 456,179 monopoles, removed 96,179 , and this leave a total sample size of 360,000 (100 monopoles times 3600 grid points) for the statistics in Figure 1. 
In summary, Figure 1 shows that the OFM method provides a nice way to analyze the field changes produced by a single point (or spherically symmetric) change in the cloud charge distribution, but because $\Delta E$ observation errors (random and/or systematic) can "rupture" this pattern, the OFM method cannot invert all cases. However, in those cases where random errors are the primary cause of such a rupture, it is possible to add artificial random errors to the simulated observations in an attempt to remove some of the random errors that are responsible for the rupture. This removal process or "monopole unmasking" is an unorthodox technique because one is combating errors by adding more errors, however we have found it to be very useful. For example, we have re-run the same monopole simulation, but this time whenever we encountered a ruptured set of simulated $\Delta E$ values, we added a random $6 \%$ error to them, and then analyzed them again using the OFM method. If the pattern was still ruptured, we again added a $6 \%$ error to the (original) simulated measurements and re-analyzed them again with the OFM method. This process was repeated (up to 100 times) to see if the OFM method could eventually find a solution. We call this process the "extended OFM method," and Figure 3 shows the results of this approach. The retrieval errors in Figure 3 are generally similar to those in Figure 1 (which indicates that adding errors can be useful in finding the hidden symmetry of monopole patterns). Whereas the standard OFM method could not analyze 96,179 (i.e., $96,179 / 456,179=21.08 \%$ ) monopoles due to ruptures, the extended OFM method had only 198 (i.e., $198 / 360198=0.05 \%$ ) that it could not analyze. 


\section{Dimensional Reduction (DR) Method}

\section{a. Model complexity versus model capability}

One can define the complexity, $n$, of a given charge model to be the number of unknown parameters that it contains. For example, the one-charge model in (2) has $n=4$ because it contains four unknown parameters $(x, y, z, Q)$. By comparison, the two-charge model in (3) has $n=8$ and is rather complex. As $n$ increases, the model must do a progressively better job of describing the $\Delta E s$ in order to obtain a "good fit" (see (7) and Table 1). If a model has the same number of parameters as there are measurements; i.e., $n=m$, the model must describe the $\Delta E S$ exactly in order to achieve a "good fit." So two disadvantages of a complex model are that it must meet stringent requirements to obtain a "good fit," and it does not obey Occam's Razor.

The capability, $\kappa$, of a particular model is the number of charge parameters that the model can describe. For example, the one- and two-charge models have $\kappa=4$ and $\kappa=8$, respectively and for these two models, $\kappa=n$. In general however, $\kappa$ need not always equal $n$. For example, the OFM method (see section 3c) can be used to reduce model complexity without reducing the model capability, and we term this approach "dimensional reduction" (DR) because the number of unknowns in the chi-square error function is reduced (see section $5 \mathrm{~b}$ for more details).

Given the above definitions, one desires to use a model that has minimum complexity, but sufficient capability. Because the one-charge model has just 4 unknown parameters, it cannot model many lightning events (e.g., intracloud discharges). Conversely, the two-charge model can describe many types of events (cloud-to-ground and intracloud discharges), but it has a high degree of complexity $(n=8)$. In between these cases, there is the 6-parameter $\left(x, y, z, p_{x}, p_{y}, p_{z}\right)$ point dipole model (Fitzgerald, 1957), the 7-parameter $\left(x, y, z, x^{\prime}, y^{\prime}, z^{\prime}, Q\right)$ 
discrete dipole model (Wilson, 1916), and the 7-parameter $\left(x, y, z, Q, p_{x}, p_{y}, p_{z}\right)$ multipole model (Koshak et al., 1999). All of these models are more capable than the one-charge model but less complex than the two-charge model.

\section{b. The DR model}

It is possible to construct a model with complexity $n=4$, yet a capability $\kappa=8$. Consider an idealized lightning flash that deposits two point charges $\mathbf{a}_{1} \equiv(x, y, z, Q)$ and $\mathbf{a}_{1}^{\prime} \equiv\left(x^{\prime}, y^{\prime}, z^{\prime}, Q^{\prime}\right)$, and that is perfectly described by the two-charge model in (3). Using (2), the $\Delta E S$ at the ground due to this flash can be written as $\Delta E_{i}=M_{1 i}\left(\mathbf{a}_{1}\right)+M_{1 i}\left(\mathbf{a}_{1}^{\prime}\right)$. Therefore, given $\mathbf{a}_{1}$ and the $\Delta E_{i}$ values, the point source $\mathbf{a}_{1}^{\prime}$ must be chosen such that $M_{1 i}\left(\mathbf{a}_{1}^{\prime}\right)=\Delta E_{i}-M_{1 i}\left(\mathbf{a}_{1}\right)$, where the difference, $\Delta E_{i}-M_{1 i}\left(\mathbf{a}_{1}\right)$, is termed the "residual." In other words, the two charges are interdependent, and one can write $\mathbf{a}_{1}^{\prime}=\left(x^{\prime}, y^{\prime}, z^{\prime}, Q^{\prime}\right)=\left(x^{\prime}\left(\mathbf{a}_{1}\right), y^{\prime}\left(\mathbf{a}_{1}\right), z^{\prime}\left(\mathbf{a}_{1}\right), Q^{\prime}\left(\mathbf{a}_{1}\right)\right)$, or in vector function notation, $\mathbf{a}_{1}^{\prime}=\mathbf{a}_{1}^{\prime}\left(\mathbf{a}_{1}\right)$. This interdependence motivates the definition of the 4-parameter "DR-model," $D_{i}\left(\mathbf{a}_{1}\right)$, given by

$$
D_{i}\left(\mathbf{a}_{1}\right)=\left\{\begin{array}{c}
M_{1 i}\left(\mathbf{a}_{1}\right)+\Upsilon_{i}\left(\mathbf{a}_{1}\right), \mathbf{a}_{1} \in V \\
\beta, \quad \mathbf{a}_{1} \notin V
\end{array},\right.
$$

where $\Upsilon\left(\mathbf{a}_{1}\right)$ is a composite function defined by

$$
\Upsilon_{i}\left(\mathbf{a}_{1}\right) \equiv M_{1 i}\left(\mathbf{a}_{1}^{\prime}\left(\mathbf{a}_{1}\right)\right)=\frac{-Q^{\prime}\left(\mathbf{a}_{1}\right) z^{\prime}\left(\mathbf{a}_{1}\right)}{2 \pi \varepsilon_{o}\left[\left(X_{i}-x^{\prime}\left(\mathbf{a}_{1}\right)\right)^{2}+\left(Y_{i}-y^{\prime}\left(\mathbf{a}_{1}\right)\right)^{2}+\left(z^{\prime}\left(\mathbf{a}_{1}\right)\right)^{2}\right]^{3 / 2}}
$$

Hence, $D_{i}\left(\mathbf{a}_{1}\right)$ represents the superposition of fields produced from two point sources, yet it contains only four unknowns. It differs from the standard two-charge model 
$M_{2 i}\left(\mathbf{a}_{2}\right)=M_{2 i}\left(\mathbf{a}_{1}, \mathbf{a}_{1}^{\prime}\right)$ given in (3) because the relationship $\mathbf{a}_{1}^{\prime}=\mathbf{a}_{1}^{\prime}\left(\mathbf{a}_{1}\right)$ has been used to reduce model complexity; section 5 c below discusses how the relationship $\mathbf{a}_{1}^{\prime}=\mathbf{a}_{1}^{\prime}\left(\mathbf{a}_{1}\right)$ is determined. Since $D_{i}\left(\mathbf{a}_{1}\right)$ is only a 4-parameter model, a good fit can be achieved when $R \cong 0.933 \sigma$, and this is easier to achieve than the value $R \cong 0.861 \sigma$ associated with the 8-parameter two-charge model $M_{2 i}\left(\mathbf{a}_{1}, \mathbf{a}_{1}^{\prime}\right.$ ) (see Table 1). Finally, $\beta$ is a very large constant that represents a physically unrealistic field change value that $D_{i}\left(\mathbf{a}_{1}\right)$ is driven to if $\mathbf{a}_{1}$ strays outside a physically reasonable vector space $V$; see section $5 \mathrm{~d}$ for further discussion on how $\mathbf{a}_{1}$ is constrained.

\section{c. Finding the optimum point sources}

In the DR method, the optimum source parameters $\mathbf{a}_{1}=(x, y, z, Q)$ are found by minimizing the reduced chi-square function

$$
\chi^{2}\left(\mathbf{a}_{1}\right)=\frac{1}{m-n} \sum_{i=1}^{m} \frac{\left(D_{i}\left(\mathbf{a}_{1}\right)-\Delta E_{i}\right)^{2}}{\sigma_{i}^{2}}
$$

We perform a grid search of a slab that intersects the negative charge region ("N-region"; Williams, 1989) as shown in Figure 4. The grid search is followed by a Powell minimization (Press et al., 1992) that iteratively adjusts the parameters in $\mathbf{a}_{1}$ until the minimum is found. If $\mathbf{a}_{1}$ strays outside the hypervolume $V$ given in (21), then the value of $\chi^{2}$ increases dramatically (i.e., $\chi^{2} \propto \beta^{2}$ for $\left.\mathbf{a}_{1} \notin V\right)$. At each step of the numerical minimization, the parameters $\left(x^{\prime}, y^{\prime}, z^{\prime}, Q^{\prime}\right)$ are retrieved using the OFM method, with the components of $\mathbf{d}$ redefined as follows

$$
d_{i} \equiv d_{i}\left(\mathbf{a}_{1}\right)=\left|\Delta E_{i}-M_{1 i}\left(\mathbf{a}_{1}\right)\right|^{-2 / 3}
$$


That is, instead of $d_{i}$ depending on $\Delta E_{i}$, it now depends on the residual $\left(\Delta E_{i}-M_{1 i}\right)$. In effect, the field change pattern is split up into two parts: one part that is described by the source $(x, y, z, Q)$, and the other part (or residual field change pattern) that is described by the source $\left(x^{\prime}, y^{\prime}, z^{\prime}, Q^{\prime}\right)$. If the residuals are of mixed polarity (or have any other "ruptures" as discussed in section 4), then $\mathbf{a}_{1}$ will not be regarded as acceptable and/or the errors in $\Delta E$ will be viewed as pathological.

Note that the DR method can also be used to effectively reduce a 3-charge (12 parameter) model down to 8 unknown parameters. In this case, $d_{i} \equiv d_{i}\left(\mathbf{a}_{2}\right)=\left|\Delta E_{i}-M_{2 i}\left(\mathbf{a}_{2}\right)\right|$, where $M_{2 i}\left(\mathbf{a}_{2}\right)$ is the two-charge model in (3). In general, the DR method can convert any $N$-charge ( $4 N$ parameter) model to a ( $4 \mathrm{~N}-4$ parameter) model by using the following definition

$$
d_{i} \equiv d_{i}\left(\mathbf{a}_{(N-1)}\right)=\left|\Delta E_{i}-\sum_{j=1}^{N-1} \frac{Q_{j} z_{j}}{2 \pi \varepsilon_{o}\left[\left(X_{i}-x_{j}\right)^{2}+\left(Y_{i}-y_{j}\right)^{2}+z_{j}^{2}\right]^{3 / 2}}\right|,
$$

where $\mathbf{a}_{(N-1)}=\left(x_{1}, y_{1}, z_{1}, Q_{1}, \ldots, x_{N-1}, y_{N-1}, z_{N-1}, Q_{N-1}\right)$.

\section{d. Constraint details}

Recall that we impose constraints on the source parameters $(x, y, z, Q)=\mathbf{a}_{1}$, where $\mathbf{a}_{1} \in V$, and $V$ is an "acceptable vector space." Our constraints are straightforward, but highly advantageous: the source location $(x, y, z)$ is restricted to the N-region of a thundercloud, and the value of $Q$ is restricted to values commonly observed or inferred for the $\mathrm{N}$-region.

Since most lightning flashes begin within or near the N-region of the thundercloud, there is little loss in generality by imposing these constraints. In fact, because the N-region usually has a small vertical extent and is less diffuse than the upper positive or P-region, and because $Q>0$ 
normally holds in the N-region, these constraints substantially reduce the size of $V$. A smaller $V$ means it is easier to minimize the chi-square function, and this translates into reduced computer time, and improved accuracy. Because it is difficult to accurately retrieve the charge parameters when the source is not located over or near the measuring network (Koshak and Krider, 1999), a horizontal range constraint can also be imposed. Such a constraint further reduces the size of $V$.

Applying these ideas, and keeping in mind the results of prior investigations (e.g., Koshak and Krider, 1989), the following constraints (on both $\mathbf{a}_{1}$ and $\mathbf{a}_{1}^{\prime}$ ) can be imposed when analyzing warm-season storms at the KSC-ER:
(a) $-30 \mathrm{~km} \leq(x$ and $y) \leq 30 \mathrm{~km}$
(b) $5 \mathrm{~km} \leq z \leq 8 \mathrm{~km}$
(c) $0<Q \leq 100 \mathrm{C}$
(d) $-30 \mathrm{~km} \leq\left(x^{\prime}\right.$ and $\left.y^{\prime}\right) \leq 30 \mathrm{~km}$
(e) $4 \mathrm{~km} \leq z^{\prime} \leq 16 \mathrm{~km}$
(f) $0<\left|Q^{\prime}\right| \leq 100 \mathrm{C}$

Again, if during the numerical minimization of the chi-square function, any element of $\mathbf{a}_{1}$ violates any of the above constraints, where $\mathbf{a}_{1}^{\prime}=\mathbf{a}_{1}^{\prime}\left(\mathbf{a}_{1}\right)$, the chi-square is assigned a large value proportional to $\beta^{2}$. The chi-square must also be assigned a large value if the residual field change pattern is ruptured (see section 4). For example, if the field changes are of mixed polarity, OFM retrievals of $\mathbf{a}_{1}^{\prime}$ will not be possible, and the chi-square must be assigned a large value.

Note that the restrictions on the altitude, $z$, in (b) above are fairly tight, but $z^{\prime}$ can vary over a much larger range, (e). This is by design because the OFM method can cover a larger altitude range more quickly and more thoroughly than a numerical minimization procedure. For example, it would not be wise to initialize the $\mathbf{a}_{1}$ source by placing it in the P-region of a 
thunderstorm because we know that this region is likely to be larger than the N-region, and therefore it would take more numerical iterations to minimize the chi-square function, all else being equal.

\section{e. Solution refinement and associated errors}

After minimizing the chi-square, a (preliminary) two-charge solution given by $\mathbf{a}_{1 c}=\left(x_{c}, y_{c}, z_{c}, Q_{c}\right)=\left(\mathbf{r}_{c}, Q_{c}\right)$ and $\mathbf{a}_{1 c}^{\prime} \equiv\left(x_{c}^{\prime}, y_{c}^{\prime}, z_{c}^{\prime}, Q_{c}^{\prime}\right)=\left(\mathbf{r}_{c}^{\prime}, Q_{c}^{\prime}\right)$ is obtained. To improve this solution, a cubical box (dimension $1 \mathrm{~km}$ ) is centered on each of the charge locations $\left(\mathbf{r}_{c}, \mathbf{r}_{c}^{\prime}\right)$. We then sample 5000 locations within each box; one sample is the center of the box, and the remaining 4999 samples are randomly selected. From each pair of sampled locations $\left(\mathbf{r}_{j}, \mathbf{r}_{j}^{\prime}\right), j=1, \ldots, 5000$, we construct the kernel matrix $\mathbf{K}_{j}=\mathbf{K}\left(\mathbf{r}_{j}, \mathbf{r}_{j}^{\prime}\right)$, and compute the standard linear least-squares charge retrieval

$$
\left[\begin{array}{l}
Q_{j} \\
Q_{j}^{\prime}
\end{array}\right]=\left(\tilde{\mathbf{K}}_{j} \mathbf{K}_{j}\right)^{-1} \tilde{\mathbf{K}}_{j} \Delta \mathbf{E} ; j=1, \ldots, 5000
$$

[see Koshak et al. (1999) for a description of the kernel matrix and linear charge retrieval theory]. Of the 5000 solutions $\left\{\left(Q_{j}, \mathbf{r}_{j}, Q_{j}^{\prime}, \mathbf{r}_{j}^{\prime}\right), j=1, \ldots, 5000\right\}$, the solution that produces a minimum in the chi-square is saved as the optimum solution. Note that $\mathbf{r}_{1} \equiv \mathbf{r}_{c}$ and $\mathbf{r}_{1}^{\prime} \equiv \mathbf{r}_{c}^{\prime}$; i.e., the value $j=1$ corresponds to the center of each box. However, $Q_{c}$ and $Q_{c}^{\prime}$ are from the chisquare minimization process using the OFM approach and therefore are not equivalent, in general, to the respective linear least-squares values $Q_{1}$ and $Q_{1}^{\prime}$.

Because a linear least-squares solution (26) has been used to find the charge values, standard linear least-squares estimation theory can be used to estimate the errors in these values. 
Specifically, the covariance matrix $\mathbf{C} \equiv\left(\tilde{\mathbf{K}} \Gamma^{-1} \mathbf{K}\right)^{-1}$ (also known as the inverse curvature matrix) contains diagonal elements that are the variances of each of the retrieved charges, and the square root of these variances provide estimates of the charge errors. Here, $\Gamma$ is a $(m \times m)$ diagonal matrix whose diagonal elements are the squared observation errors $\sigma_{i}^{2}, i=1, \ldots, m$. Of course, error estimates derived from $\mathbf{C}$ are only valid if the charge locations themselves are reasonably accurate (and the model is perfect).

\section{Application of the DR Method}

We have used the DR method to analyze a 30 minute interval (20:30-21:00 UTC) of a warm-season Florida thunderstorm that occurred at the KSC-ER on 21 September 1998. Sensors $\# 1$ and \#32 were faulty during this period and were not included in the analysis. Flashes that produced a $\Delta E>100 \mathrm{~V} / \mathrm{m}$ at 2 or more sites were analyzed; i.e., a total of 154 flashes. Of these, 115 flashes produced a reduced chi-square less than 5 ; the average reduced chi-square for these 115 solutions was 1.058 with a standard deviation of 1.017 . [We used a chi-square threshold of 5 because there is uncertainty in the value of $\sigma$ in (4), and because many solutions meeting this criterion were physically reasonable.]

The altitudes of the 115 charge solutions (large circles) are plotted in Figure 5 together with LDAR data (small dots). Positive charge deposition is denoted with red circles, and negative deposition with blue. Larger circles indicate greater amounts of charge deposition; i.e., the radius $r$ of each circle has been scaled to the vertical axis (see Koshak and Krider, 1989) and is a solution to the breakdown field equation $E_{b}=Q /\left(4 \pi \varepsilon_{o} r^{2}\right)$ with $E_{b}=400 \mathrm{kV} / \mathrm{m}$. The LDAR system maps the source locations of the VHF radio impulses produced by both intracloud (IC) and cloud-to-ground (CG) lightning with a time-resolution of about $100 \mu$ s and a spatial accuracy 
of hundreds of meters or less for flashes that are within or near the perimeter of the KSC-ER field mill network (Maier et al., 1995). For lightning that is within about $40 \mathrm{~km}$ of the KSC-ER, the LDAR location accuracy is better than $1 \mathrm{~km}$ (see Boccippio et al., 2001, Parts 1 and 2 for additional details on LDAR performance). The LDAR data are color-coded according to time in the plot projection.

To understand the quality of the charge solutions in Figure 5, we have examined individual plots of each flash to see how well the lightning charge locations and the LDAR data agree. Given that the $\Delta E$ and LDAR data are completely independent datasets, it was encouraging to find that a high percentage of the model solutions had $(x, y)$ locations that were within $1 \mathrm{~km}$ of, or co-aligned with, the $(x, y)$ positions of LDAR sources. Overall, we found that there were three types of solutions: (I) those that lined up well with LDAR sources in $x, y$, and $z$, (II) those that lined up well in $x$ and $y$ but had offsets in $z$, and (III) those that had offsets in $x$, $y$, and $z$.

Figures 6, 7, and 8 show examples of solution types I, II and III, respectively. Most offsets in $z$ (Figures 7 and 8) were caused by a fundamental "dipole ambiguity." For example, consider a vertically oriented cloud discharge that produces a dipole moment change $(20 \mathrm{C})(3 \mathrm{~km})$ $=60 \mathrm{C}-\mathrm{km}$. If the charge transfer is reduced to $15 \mathrm{C}$ and the discharge length is increased to $4 \mathrm{~km}$, the dipole moment does not change; i.e., $(15 \mathrm{C})(4 \mathrm{~km})=60 \mathrm{Ckm}$, and it is possible (depending on source location and network geometry) that the two $\Delta E$ patterns associated with these two sources are nearly indistinguishable. Moreover, any small features that distinguish the patterns can be completely removed by errors in $\Delta E$. Hence, fundamental ambiguities arise whenever different dipole sources effectively produce the same set of $\Delta E$ values. For all the solutions in Figures 6-8, the charge retrieval errors (i.e. the square root of the diagonal elements of the 
covariance matrix $\mathbf{C}$ ) were typically less than $1 \mathrm{C}$. Of course, this magnitude of error applies primarily to type I (Figure 6) where the charge locations are reasonably accurate and the linear least squares error estimation theory can be used.

Of the 115 flashes, $21(18.3 \%)$ were exceptional fits (type I), $61(53.0 \%)$ could be fit reasonably well (type II), and $25(21.7 \%)$ were type III. Additionally, 5 (4.3\%) were complicated flashes that were too difficult to categorize into any one of the three main types, and $3(2.6 \%)$ were false triggers in the electric field dataset (i.e. the $\Delta E s$ were not associated with LDAR events). Since in this case study a total of $82(71.3 \%)$ could be fit either reasonably or exceptionally well, we believe that the DR method is a valuable tool for describing lightning field changes.

In section 7 to follow, we will describe how we use LDAR data to constrain the charge location model parameters so that, by definition, they do not contain offsets in position. These charge retrievals will then be compared to both satellite- and ground-based lightning observations in section 8 .

\section{LDAR-Constrained (LC) Method}

Because lightning effectively deposits charge within thundercloud charge centers and because LDAR traces the geometrical development of the lightning channel with high precision, the LDAR data provides an ideal constraint for finding the best model charge solutions. In particular, LDAR data can be used to help determine both the horizontal and vertical positions of the model charges, thereby eliminating dipole ambiguities.

The LDAR-constrained (LC) charge retrieval method is straightforward. LDAR data for individual flashes are displayed on the computer monitor, and a mouse is used to click the 
expected locations of the charge centers (usually the endpoints of the LDAR-mapped lightning channel). For example, a typical vertical or slanted cloud discharge between the N- and Pregions would receive two mouse clicks, one at the lower end of the channel within the N-region, and one at the upper end within the P-region. Calling these endpoint locations $\mathbf{r}_{1}$ and $\mathbf{r}_{1}^{\prime}$, respectively, we center a cubical box (dimension $1 \mathrm{~km}$ ) about each of these locations and internally sample the box volumes exactly as described in section $5 \mathrm{e}$. That is, we compute 5000 solutions $\left\{\left(Q_{j}, \mathbf{r}_{j}, Q_{j}^{\prime}, \mathbf{r}_{j}^{\prime}\right), j=1, \ldots, 5000\right\}$, and the solution that produces a minimum in the chisquare is saved as the optimum solution. Hence, the discharge orientation is obviously tightly constrained by the LDAR channel endpoints, but the model charges are allowed to deviate a bit from these endpoints in order to obtain a better fit to the $\Delta E$ observations.

\section{Satellite-Observed Flashes}

The results of the LC method have been compared to the locations of optical pulses detected by the Lightning Imaging Sensor (LIS) on the low Earth-orbiting Tropical Rainfall Measuring Mission (TRMM) satellite (Christian et al., 1999). Several flashes from different storms that occurred near the KSC-ER have been studied in detail. To augment this investigation, we have also compared both datasets to data provided by the U.S. National Lightning Detection Network $^{\mathrm{TM}}$ (NLDN) and the Cloud-to-Ground (CG) Lightning Surveillance System (CGLSS) that covers the region of the KSC-ER. Of course, the charge solutions have also been compared to the LDAR data that have been used to constrain the charge locations. The CGLSS and NLDN systems locate the ground strike points of the individual return strokes in CG flashes with an accuracy of a few hundred meters (see, for example, Cummins et al., 1998a,b). Also, Thomas et 
al. (2000) have found good overall agreement between Lightning Mapping Array (LMA, a system similar to LDAR) sources and LIS flash locations in Oklahoma.

Although it is rather rare to have a TRMM overpass at the same time a thunderstorm is in progress at the KSC-ER, 7 such periods have been identified within the years 1998-1999 and were analyzed for this study (see Table 2). Each overpass lasted from 2 to 3 minutes, and the total observation time was 15 minutes. The KSC-ER field mill (FM) network detected a total of 94 discharges (each producing $\Delta E>100 \mathrm{~V} / \mathrm{m}$ at 2 or more FM sites) during this 15 minute period, and the LDAR system detected all 94 of these discharges. Figure 9 shows examples of two overpasses from the unique perspective of the LIS low-Earth orbit; both the LIS background image in the near infrared and the density of LIS-detected flashes are provided.

The CGLSS data were not available for days 180,219 , and 229 , but when they were available, there were always more CG events in the CGLSS dataset than were detected by the NLDN. Also, many of the 94 discharges were cloud flashes that were not reported by the CGLSS or NLDN unless they were incorrectly classified.

The lightning flashes that were not reported by LIS were divided into two categories, a Legitimate Miss (LM) or a Not Processed (NP) type. Typically, the LMs were due to LIS data post-filtering, "pixel splitting" of radiance, and/or cloud attenuation. The NPs were due to LIS buffer overflows, flashes (just) outside LIS field-of-view, and spacecraft incidents (e.g. telemetry bit errors, and spacecraft attitude maneuvers). Of the 94 discharges in our case study, 93 appear to be regular "flashes," and one was a small breakdown event that preceded a normal CG flash. Of the 93 flashes, LIS did not report 17, and of these, we estimate that 6 were LMs, and 11 were NPs. The NPs were mostly due to buffer overflows and/or outside field-of-view cases, although one flash was probably lost due to a bit error in the spacecraft telemetry. 
Lightning charges were computed for all 94 discharges and compared to the patterns of $\Delta E$ obtained from the field mill network, the spatial-temporal development of the LDAR sources, with the locations of LIS optical events, and with the NLDN and CGLSS ground strike points, when available. As expected, the LDAR-constrained charge retrievals had locations that were in very good agreement with data obtained from the other lightning detection systems. With an assumed $\sigma$ equal to $15 \%$ of the typical (rms) field change value, the median and average reduced chi-square for the 94 flashes was 3.69 and 9.54 , respectively. A total of $55(\sim 60 \%)$ of the 94 flashes had reduced chi-square values less than 5, and were good solutions. We also found physically reasonable solutions for larger reduced chi-square values (see Figures 11 and 13 to follow). Additionally, it is important to note that when one adds external constraints (e.g. LDAR data) to the retrieval process, it is common to have an increase in the chi-square value. This is because the (now constrained) model parameters can no longer be adjusted in any way desired to mathematically minimize the chi-square. Therefore, when applying valid external constraints (such as LDAR data), it is incorrect to infer that any increases in chi-square imply a lower quality solution and/or a physically unrealistic model, especially when $\Delta E$ errors are actually large when assumed to be reasonably small.

Figures 10-15 show detailed results for 6 of the 94 discharges that were analyzed using the LC method. The red and blue circles show the locations of the positive and negative lightning-charges, $Q$ and $Q^{\prime}$, respectively, that the lightning effectively deposited in the thundercloud. The values of $Q$ and $Q^{\prime}$ are given at the top of each plot in Coulombs. The curved lines in the plan views on the lower left show the contours of $\Delta E$ in $\mathrm{V} / \mathrm{m}$; red contours indicate positive values, and blue contours indicate negative values. The reduced chi-square values associated with the solutions in Figures 10-15 are, respectively: 1.82, 11.8, 0.93, 6.55, 
0.79 , and 4.4. The panels on the upper right show plots of altitude versus time (in GMT) with color coding; the total time is 2 seconds in each plot except for Figure 11 which is 1 second. The large squares show the $(x, y)$ locations of the LIS optical events, and the large diamonds are the LIS flash locations. Since the LIS sensor does not directly observe the altitude of a flash, the optical events and locations are placed near the top of each altitude cross section plot for optimum readability, and to remind the reader that these data are derived from a satellite that is viewing the KSC-ER from above. The small dots show the locations of LDAR sources, and each dot has been color-coded with respect to time using the same color sequence as in Figure 5 . The small squares show the locations of the first LDAR source (black) and last (orange) in the discharge. The black and orange " $\mathrm{X}$ " symbols show the locations of negative ground strike points from NLDN and CGLSS, respectively; a "+" symbol indicates that the ground flash had a positive polarity.

The discharges in Figures 10-15 were selected because they include both CG and cloud flashes, and because they occur to the north (Figure 10), south (Figure 11), east (Figure 12), west (Figure 13), and over (Figure 14) the network. The discharge in Figure 14 is particularly interesting because it is a small breakdown event that precedes a CG flash. Even though the flash types and locations are diverse, the LDAR dataset provides good location constraints so that the linear least-square charge retrievals are physically reasonable in both magnitude and polarity.

We believe that the discharge in Figure 11 is a cloud flash, rather than a positive CG as indicated by NLDN/CGLSS. This belief is supported by several factors: (1) the charge retrievals are roughly equal and opposite; i.e., a $10 \%$ charge retrieval error is possible ${ }^{*}$, (2) no LDAR

${ }^{*}$ Taking the charge values literally implies that almost $4 \mathrm{C}(40.070 \mathrm{C}-36.123 \mathrm{C})$ of negative charge is deposited to ground, thereby contradicting the polarity inferred by NLDN/CGLSS. 
sources are below about $5 \mathrm{~km}$, and (3) there are similarities in the field change characteristics of positive CGs and normal vertical cloud flashes. Previous investigators have recommended classifying positive flashes with peak currents less than $10 \mathrm{kA}$ as cloud discharges [Cummins et al., 1998b; Zajac and Rutledge, 2001]; and a $15 \mathrm{kA}$ threshold has been more recently used (Cummins personal communication, 2006). Since the peak currents inferred by NLDN and CGLSS are 15.3, and 14.0 kA, respectively, for the flash in Figure 11, this is one example where application of a simple $10 \mathrm{kA}$ threshold criterion would appear inadequate.

An analysis of the LIS raw data shows that the LIS instrument likely detected the small discharge in Figure 14, but that it was later removed by the LIS data processing algorithm. Because this discharge was less than $0.9 \mathrm{~s}$ before, and within $10 \mathrm{~km}$ of, the $\mathrm{CG}$ flash provided in Figure 15 , we think that these discharges might be physically correlated to one another. From this point of view, the small discharge in Figure 14 is not considered a "legitimate flash" on its own, and one can argue that LIS properly reported just the primary portion of the overall phenomenon.

\section{Summary}

We have introduced an Overdetermined Fixed Matrix (OFM) method for finding the optimum parameters of a 4-parameter Q-model. This method offers two distinct advantages over a previous 4-station solution scheme: first, one can use more than four $\Delta E$ observations to obtain a highly constrained source retrieval. Second, the inverse matrix is "fixed" which means it does not depend on the values of $\Delta E$ (as in the previous 4-station solution scheme), and hence the matrix does not change from flash to flash. Because the matrix only depends on the measurement network geometry, only one matrix inversion is required for any given network. 
This speeds up computations, and it also allows one to more thoroughly understand the retrieval errors associated with any particular network geometry since the eigenvalues of a fixed matrix are fixed (see Chapter 6 of Twomey, 1977 regarding the importance of eigenanalyses in linear retrievals).

We have shown that the OFM method accurately retrieves the parameters of simulated, single-charge sources in the presence of $\Delta E$ errors. For $25 \mathrm{C}$ monopoles placed $6.5 \mathrm{~km}$ over the field mill network at the KSC-ER, the horizontal $(x, y)$ and vertical $(z)$ retrieval errors were each less than $0.5 \mathrm{~km}$ and the charge retrieval error was less than $1 \mathrm{C}$. As with most analysis methods, retrieval errors increase for sources located off the network, and continue to increase with source range.

Most importantly, the OFM method has been used to reduce the complexity (number of model parameters) of a two-charge model. This "dimensional reduction" (DR) approach significantly reduces the computational burden because the number of unknown parameters in the chi-square function is reduced from 8 to just 4 . Because of this reduction in dimension, there is a reduced chance of getting "stuck" in a local minimum of the chi-square function (because there are fewer unknowns), and consequently the DR method has the potential to significantly reduce retrieval errors.

One should note that the DR method is not simply a practical approach for solving charge retrieval problems. Rather, it introduces a fundamental, yet subtle, aspect of lightning charge modeling: the distinction between "model complexity" and "model capability." Model complexity is the number of unknown charge parameters contained in the model, whereas model capability is the number of charge parameters the model can describe. [For example, the model provided in (21) has 4 unknown charge parameters (complexity $=4$ ), but it can describe 8 charge 
parameters (capability $=8$ ).] We also showed that when the complexity of a model increases, one is forced to model the $\Delta E s$ more accurately in order to obtain a good fit (see (7) and Table 1). Since the DR method reduces model complexity but maintains model capability, it makes it easier to obtain a good fit. As an added benefit, the DR method also satisfies the simplicity principle of Occam's Razor.

Finally, we have used the DR method to analyze over one-hundred lightning flashes in a Florida thunderstorm, and it produced a high percentage of physically reasonable solutions, as judged by comparisons with LDAR data. Unfortunately, several DR solutions suffered from a fundamental "dipole ambiguity" that produces vertical offsets in the retrieved charge altitudes relative to those that are inferred from the LDAR dataset. This problem has been solved by using LDAR data to constrain the location of the lightning charges, and the resulting "LDARconstrained" charge solutions have been compared to LIS optical sources and the locations of the ground strike points obtained from the NLDN and the CGLSS at the KSC-ER. We have given examples of these comparisons for several Florida thunderstorms, and in the future we expect that additional comparisons will provide relationships between lightning charges and the optical radiance, the number of LDAR sources, and in the case of CG flashes, the peak current and multiplicity.

\section{Acknowledgements}

This research has been supported by the NASA Headquarters Validation Program, NRA 97-MTPE-03, under the direction of David O'C. Starr, by the NASA Earth Observing System (EOS) program under task \#621-1598, and by the NASA Kennedy Space Center, Grant NAG 10302. We also appreciate the assistance of Mr. Johnny Hall (Computer Sciences Corp.) in 
obtaining graphic images of the LIS overpasses over the KSC region, and the many helpful discussions with Dr. Richard Blakeslee regarding the occurrence of cloud flashes in NLDN data. 


\section{REFERENCES}

Bevington, P. R. and D. K. Robinson, 2003: Data reduction and error analysis for the physical sciences, $3^{\text {rd }}$ Edition McGraw-Hill, New York.

Boccippio, D. J., S. Heckman, S. J. Goodman, 2001: A diagnostic analysis of the Kennedy Space Center LDAR network. 1. Data characteristics, J. Geophys. Res., 106, 4769-4786.

Boccippio, D. J., S. Heckman, S. J. Goodman, 2001: A diagnostic analysis of the Kennedy Space Center LDAR network. 2. Cross-sensor studies, J. Geophys. Res., 106, 4787-4796.

Christian, H. J., R. J. Blakeslee, S. J. Goodman, D. A. Mach, M. F. Stewart, D. E. Buechler, W. J. Koshak, J. M. Hall, W. L. Boeck, K. T. Driscoll, and D. J. Boccippio, 1999: The Lightning Imaging Sensor, Proc. $11^{\text {th }}$ Int. Conf. on Atmospheric Electricity, Guntersville, AL, International Commission on Atmospheric Electricity, 746-749.

Cummins, K. L., E. P. Krider, and M. D. Malone, 1998a: The U.S. National Lightning Detection Network $^{\mathrm{TM}}$ and applications of cloud-to-ground lightning data by electric power utilities, IEEE Trans. on Electro. Comp., 40, 465-480.

Cummins, K. L., M. J. Murphy, E. A. Bardo, W. L. Hiscox, R. B. Pyle, and A. E. Pifer, 1998b: A combined TOA/MDF technology upgrade of the U.S. National Lightning Detection Network, J. Geophys. Res., 103, 9035-9044. 
Fitzgerald, D. R., 1957: Some theoretical aspects of the relation of surface electric field observations to cloud charge distribution, J. Meteorol., 14, 505-512.

Jacobson, E. A., and E. P. Krider, 1976: Electrostatic field changes produced by Florida lightning, J. Atmos. Sci., 33, 103-117.

Koshak, W. J., and E. P. Krider, 1989: Analysis of lightning field changes during active Florida thunderstorms, J. Geophys. Res., 94, 1165-1186.

Koshak, W. J., 1991: Analysis of lightning field changes produced by Florida thunderstorms, NASA Tech. Memo \#103539, April.

Koshak, W. J., and E. P. Krider, 1994: A linear method for analyzing lightning field changes, $J$. Atmos. Sci., 51, 473-488.

Koshak, W. J., E. P. Krider, and M. J. Murphy, 1999: A multipole expansion method for analyzing lightning field changes, J. Geophys. Res., 104, 9617-9633.

Krehbiel, P. R., M. Brook, and R. A. McCrory, 1979: An analysis of the charge structure of lightning discharges to ground, J. Geophys. Res., 84, 2432-2456.

Krehbiel, P. R., 1981: An analysis of the electric field change produced by lightning. Ph.D. thesis, Univ. of Manchester Inst. of Sci. and Technol., Manchester, England. 
Krehbiel, P. R., 1986: The electrical structure of thunderstorms, in The Earth's Electrical Environment, Chap. 8, Natl. Acad. of Sci. Press, Washington, D.C.

Maier, L. M., and E. P. Krider, 1986: The charges that are deposited by cloud-to-ground lightning in Florida, J. Geophys. Res., 91, 13 275-13 289.

Maier, L. M., C. Lennon, T. Britt, S. Schaefer, 1995: Lightning Detection And Ranging (LDAR) system performance analysis, Proceedings on the $6^{\text {th }}$ Conf. on Aviation Weather Systems, AMS Meeting, 15-20 January 1995, Dallas, Texas.

Murphy, M. J., E. P. Krider, and M. W. Maier, 1996: Lightning charge analyses in small Convection and Precipitation Experiment (CaPE) storms, J. Geophys. Res., 101, 29 615-29 626.

Press, W. H., W. T. Vetterling, S. A. Teukolsky, and B. P. Flannery, 1992: Numerical Recipes in Fortran 77, The Art of Scientific Computing, $2^{\text {nd }}$ Edition, Cambridge University Press, New York.

Thomas, R. J., P. R. Krehbiel, W. Rison, T. Hamlin, D. J. Boccippio, S. J. Goodman, H. J. Christian, 2000: Comparison of ground-based 3-dimensional lightning mapping observations with satellite-based LIS observations in Oklahoma, Geophys. Res. Letters, 27, 1703-1706.

Twomey, S. A., 1977: Introduction to the Mathematics of Inversion in Remote Sensing and Indirect Measurements, Elsevier, New York. 
Williams, E. R., 1989: The tripole structure of thunderstorms, J. Geophys. Res., 94, 13151-13167.

Wilson, C. T. R., 1916: On some determinations of the sign and magnitude of electric discharges in lightning flashes, Proc. R. Meteorol. Soc., Ser. A, 92, 555-574.

Wilson, C. T. R., 1920: Investigations on lightning discharges and on the electric field of thunderstorms, Phil. Trans. R. Soc. Ser. A, 221, 73-115.

Workman, E. J., R. E. Holzer, G. T. Pelsor, 1942: The electrical structure of thunderstorms, National Advisory Committee for Aeronautics (NACA) Tech. Note 864, 47 pp.

Zajac, B. A., S. A. Rutledge, 2001: Cloud-to-ground lightning activity in the contiguous United States from 1995 to 1999, Mon. Weather Rev., 129, 999-1019. 


\section{Figure Caption List}

Fig. 1. Monte Carlo error analyses of monopole sources retrieved with the OFM approach showing (a) average percent $\Delta E$ modeling error, (b) rms horizontal distance error, (c) rms altitude errors, and (d) rms charge errors. The color key corresponding to these figures is shown in Figure 2. The error statistics are derived for 100 randomized sources at each grid point, with a grid resolution of $1 \mathrm{~km}$.

Fig. 2. The key applied to Figure 1 (and Figure 3 to follow) that associates color to error values for the three error categories shown.

Fig. 3. Same type of error analysis as in Figure 1 except that a $6 \%$ random error was purposely added to the simulated $\Delta E S$ (in several independent trials) in order to nullify some of the simulated observation errors. This resulted in significantly increasing the number of monopole sources successfully modeled.

Fig. 4. A summary of Dimensional Reduction (DR) where eight unknowns $\left(x, y, z, Q, x^{\prime}, y^{\prime}, z^{\prime}, Q^{\prime}\right)$ are converted to just four $(x, y, z, Q)$ using the OFM method. An example of one of the residuals is shown in purple. As the chi square function (23) is minimized, the source, $\mathbf{a}_{1}$ (which produces field $M_{1 i}\left(\mathbf{a}_{1}\right)$ at the ground), approaches both the location and magnitude of the lower thundercloud charge, and the OFM-retrieved source, $\mathbf{a}_{1}^{\prime}$, approaches the location and magnitude of the upper thundercloud charge. 
Fig. 5. Altitude vs. time plot of the DR-retrieved charges and associated LDAR data for a 30 minute period of a thunderstorm that occurred on 21 September 1998. The red circles indicate positive changes in cloud charge, and the blue circles indicate negative changes. The small dots are the LDAR radio pulses (color coded with time). See Figures 6-8 below for further details on individual flashes.

Fig. 6. Examples of charge solutions derived from the DR method (and picked from the results in Figure 5) that compare well with LDAR data.

Fig. 7. Examples of charge solutions derived from the DR method (and picked from the results in Figure 5) that show charge location offset errors in the vertical relative to LDAR data.

Fig. 8. Examples of flashes derived from the DR method (and picked from the results in Figure 5) that show charge location offset errors in the horizontal and vertical relative to LDAR data.

Fig. 9. Examples of two LIS overpasses of KSC on (a) 14 May 1999, and (b) 21 September 1998. The background cloud image is not always collected if lightning rates are high. A total of 7 overpasses (on seven different storm days) were analyzed in this study.

Fig. 10. A CG flash located north of the KSC-ER FM network. The red and blue circles denote lightning-caused changes in thundercloud charge given by $Q$ and $Q^{\prime}$, respectively as derived by using LDAR constraints and linear least squares theory. The values of $Q$ and $Q^{\prime}$ in coulombs are given at the top of the plot. The large squares are individual LIS optical events and the large 
diamond is the LIS-derived flash location. The small dots indicate VHF source locations for the flash derived from the LDAR network; the small squares identify what VHF source occurred first (black) and last (orange) within the flash. The black and orange "Xs" mark the NLDN- and CGLSS- derived CG location, respectively.

Fig. 11. An IC flash located well south of the KSC-ER network. See caption of Figure 10 for a description of the plot symbols. We believe this flash was incorrectly categorized as a positive CG by the NLDN and CGLSS systems (see main text for a discussion).

Fig. 12. A CG flash (with IC component) located east of the KSC-ER network. See caption of Figure 10 for a description of the plot symbols.

Fig. 13. An IC flash located slightly west of the KSC-ER network. See caption of Figure 10 for a description of the plot symbols.

Fig. 14. A small discharge over the KSC-ER network that the LIS instrument detected, but that the LIS data processing algorithm removed. It precedes the CG shown in Figure 15 by less than $0.9 \mathrm{~s}$, and is also within about $10 \mathrm{~km}$ of the CG (see main text for more comments). The CGLSS and NLDN ground strike locations shown are associated with the CG that follows, but appear in this plot due to sensor timing errors. See caption of Figure 10 for a description of the plot symbols. 
Fig. 15. The CG that quickly follows, and is likely correlated with, the small discharge shown in Figure 14. The CGLSS and NLDN ground strike locations for this CG appear in Figure 14 due to sensor timing errors. See caption of Figure 10 for a description of the plot symbols. 


\section{Values of the Coefficient $\xi$}

Table 1. The numerical value of the coefficient $\xi$ in equation (7) as a function of model complexity $n$, when $m=31$ sensors. The bolded values indicate the customary 1 - and 2- charge models.

\begin{tabular}{lc}
\hline$n$ & $\xi(n ; m=31)$ \\
\hline 1 & 0.984 \\
2 & 0.967 \\
3 & 0.950 \\
$\mathbf{4}$ (e.g., the 1-charge model) & $\mathbf{0 . 9 3 3}$ \\
5 & 0.916 \\
6 & 0.898 \\
7 & 0.880 \\
$\mathbf{8}$ (e.g., the 2-charge model) & $\mathbf{0 . 8 6 1}$ \\
9 & 0.842 \\
10 & 0.823 \\
11 & 0.803 \\
12 & 0.783 \\
13 & 0.762 \\
14 & 0.741 \\
15 & 0.718 \\
16 & 0.696 \\
17 & 0.672 \\
18 & 0.648 \\
19 & 0.622 \\
20 & 0.596 \\
21 & 0.568 \\
22 & 0.539 \\
23 & 0.508 \\
24 & 0.475 \\
25 & 0.440 \\
26 & 0.402 \\
27 & 0.359 \\
28 & 0.311 \\
29 & 0.254 \\
30 & 0.180 \\
31 & 0.000 \\
\hline
\end{tabular}




\section{Summary of Storm Periods}

Table 2. Summary of the Florida storm periods analyzed that were associated with LIS overpasses of the KSC-ER.

\begin{tabular}{ccccc}
\hline Date & Julian Day & Time (GMT) & $\begin{array}{c}\text { \# flashes } \\
\text { (FM-derived) }\end{array}$ & $\begin{array}{c}\text { \# Bad } \\
\text { FMs }\end{array}$ \\
& & & & \\
\hline 21 Sep 1998 & 264 & $20: 39-20: 42$ & 13 & 2 \\
08 May 1999 & 128 & $22: 04-22: 06$ & 11 & 1 \\
14 May 1999 & 134 & $19: 38-19: 40$ & 22 & 3 \\
11 Jun 1999 & 162 & $05: 06-05: 08$ & 14 & 1 \\
29 Jun 1999 & 180 & $19: 01-19: 03$ & 21 & 0 \\
07 Aug 1999 & 219 & $23: 37-23: 39$ & 7 & 0 \\
17 Aug 1999 & 229 & $17: 58-18: 00$ & 6 & Total =94 \\
\hline
\end{tabular}



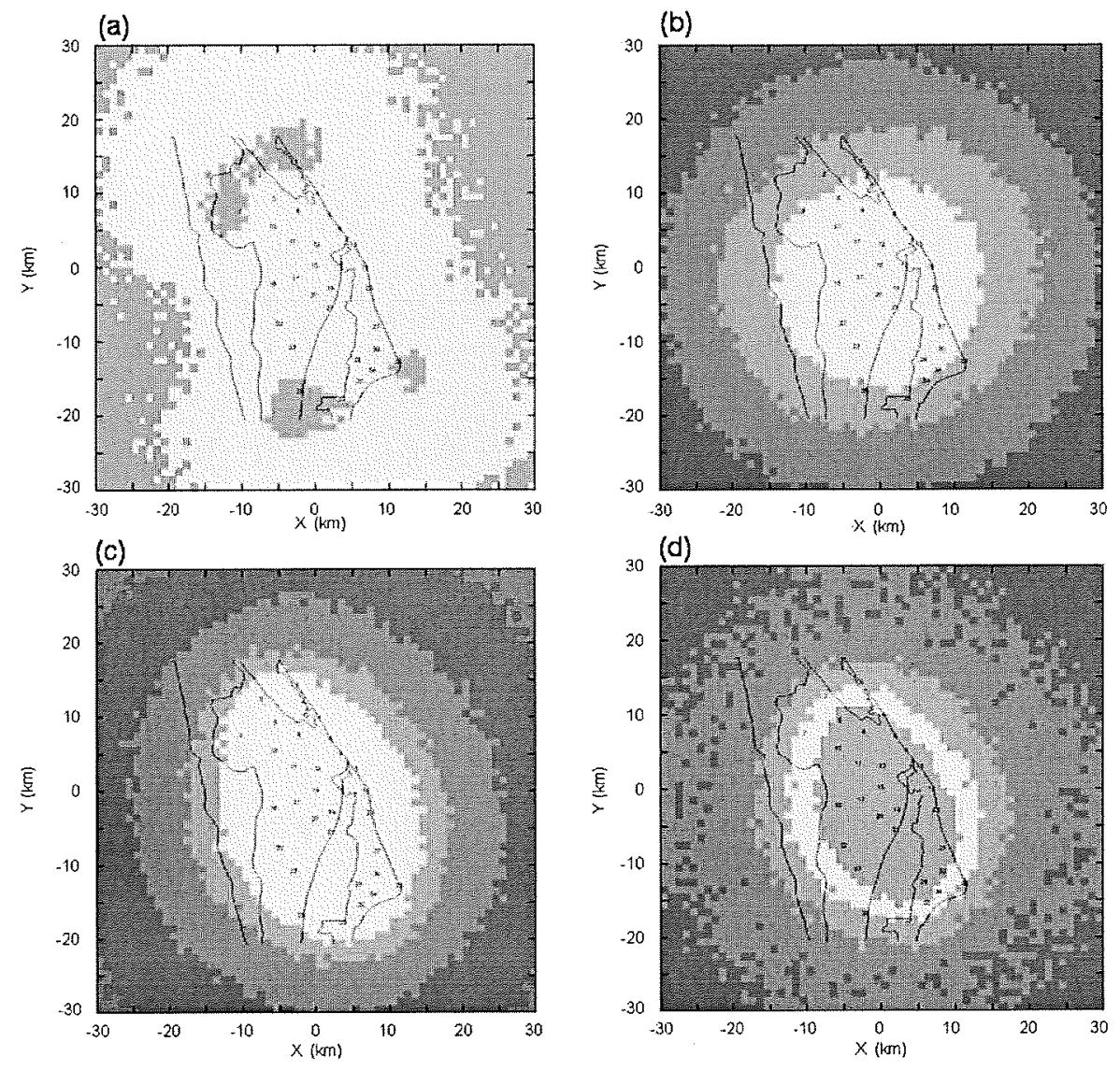

Fig. 1. Monte Carlo error analyses of monopole sources retrieved with the OFM approach showing (a) average percent $\Delta E$ modeling error, (b) rms horizontal distance error, (c) rms altitude errors, and (d) rms charge errors. The color key corresponding to these figures is shown in Figure 2. The error statistics are derived for 100 randomized sources at each grid point, with a grid resolution of $1 \mathrm{~km}$. 


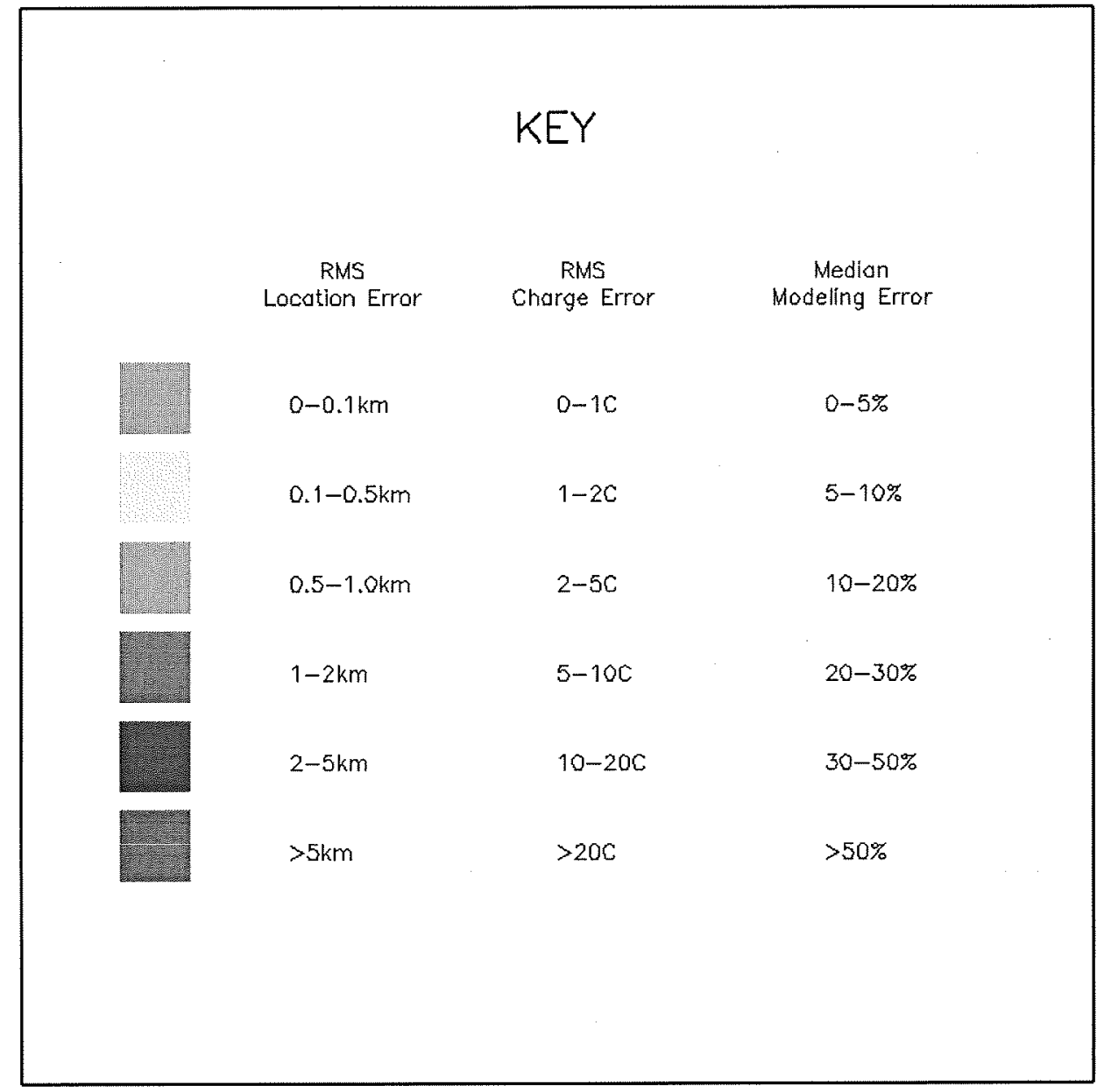

Fig. 2. The key applied to Figure 1 (and Figure 3 to follow) that associates color to error values for the three error categories shown. 
(a)
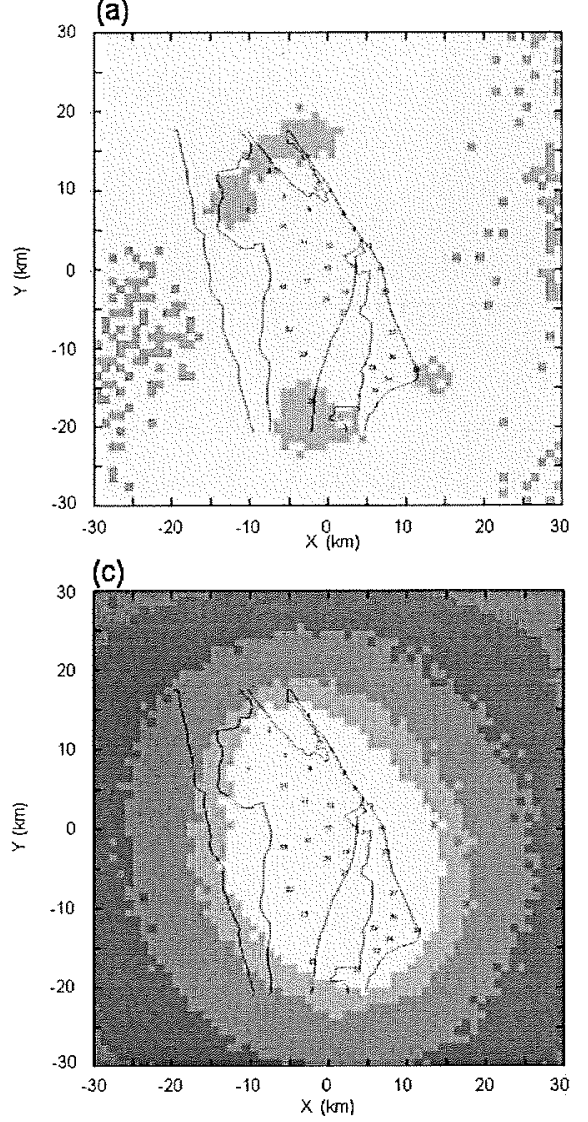

(b)
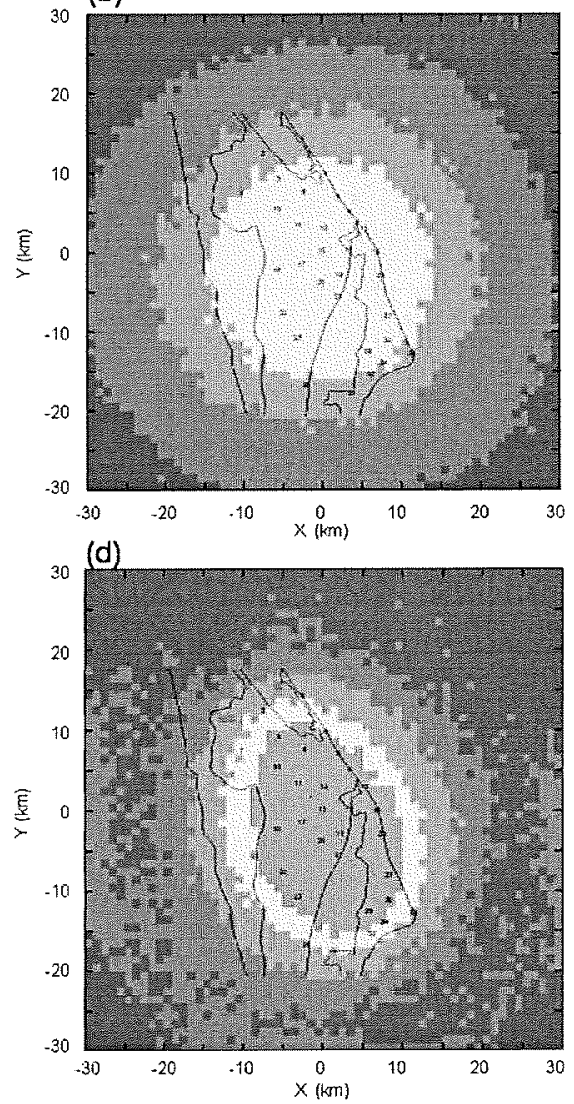

Fig. 3. Same type of error analysis as in Figure 1 except that a $6 \%$ random error was purposely added to the simulated $\Delta E s$ (in several independent trials) in order to nullify some of the simulated observation errors. This resulted in significantly increasing the number of monopole sources successfully modeled. 


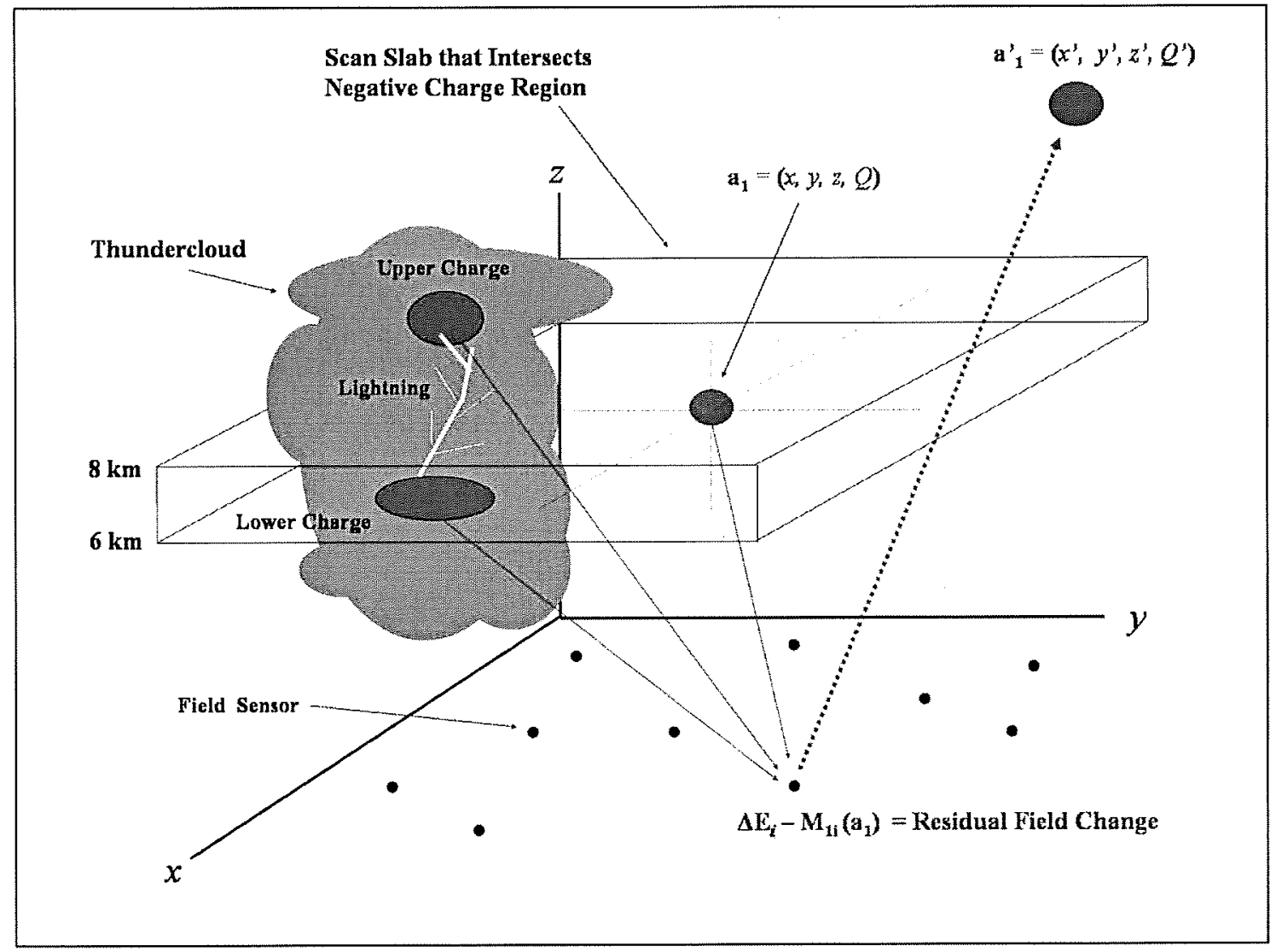

Fig. 4. A summary of Dimensional Reduction (DR) where eight unknowns $\left(x, y, z, Q, x^{\prime}, y^{\prime}, z^{\prime}, Q^{\prime}\right)$ are converted to just four $(x, y, z, Q)$ using the OFM method. An example of one of the residuals is shown in purple. As the chi square function (23) is minimized, the source, $\mathbf{a}_{1}$ (which produces field $M_{1 i}\left(\mathbf{a}_{1}\right)$ at the ground), approaches both the location and magnitude of the lower thundercloud charge, and the OFM-retrieved source, $\mathbf{a}_{1}^{\prime}$, approaches the location and magnitude of the upper thundercloud charge. 


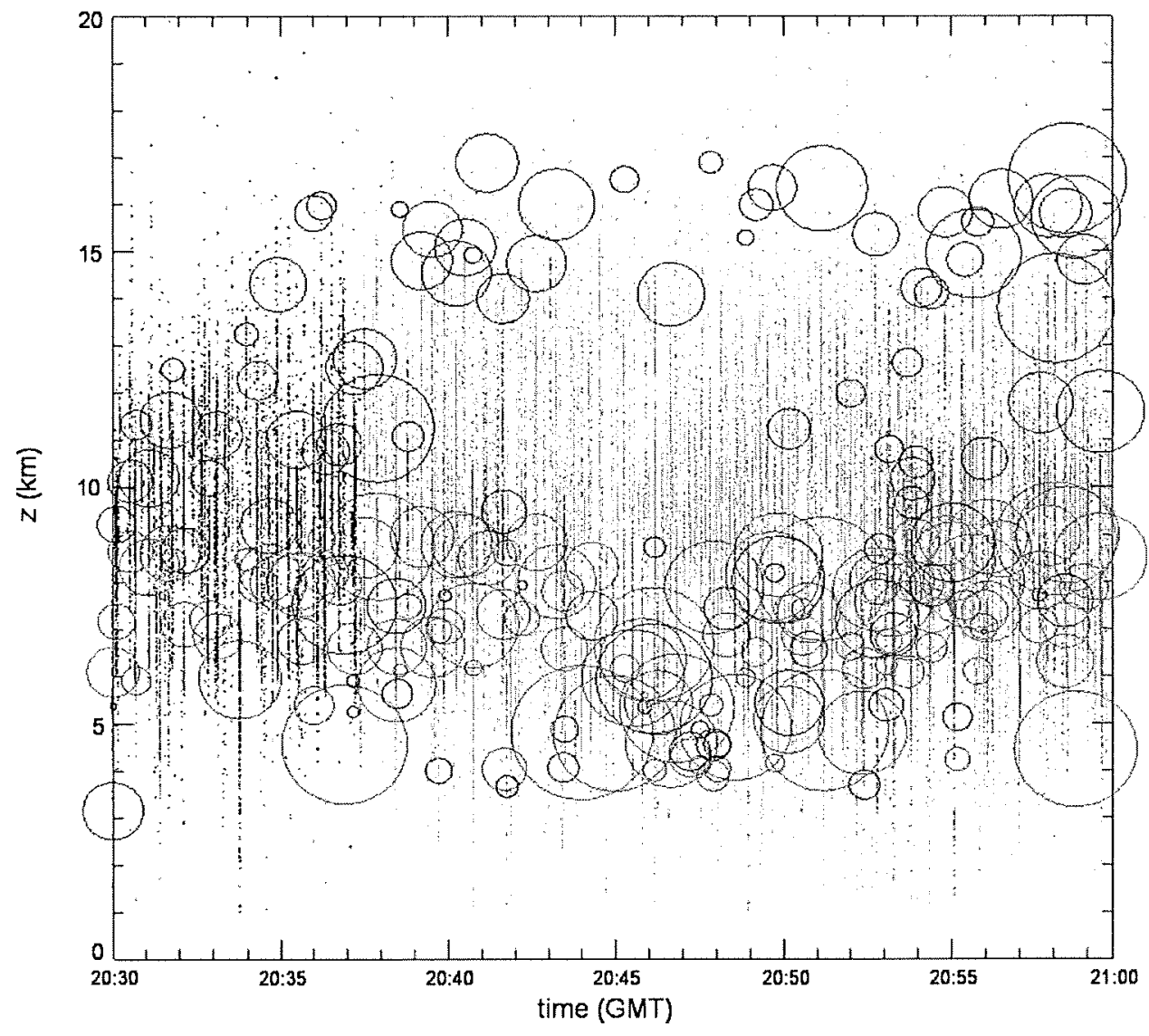

Fig. 5. Altitude vs. time plot of the DR-retrieved charges and associated LDAR data for a 30 minute period of a thunderstorm that occurred on 21 September 1998. The red circles indicate positive changes in cloud charge, and the blue circles indicate negative changes. The small dots are the LDAR radio pulses (color coded with time). See Figures 6-8 below for further details on individual flashes. 
(a)

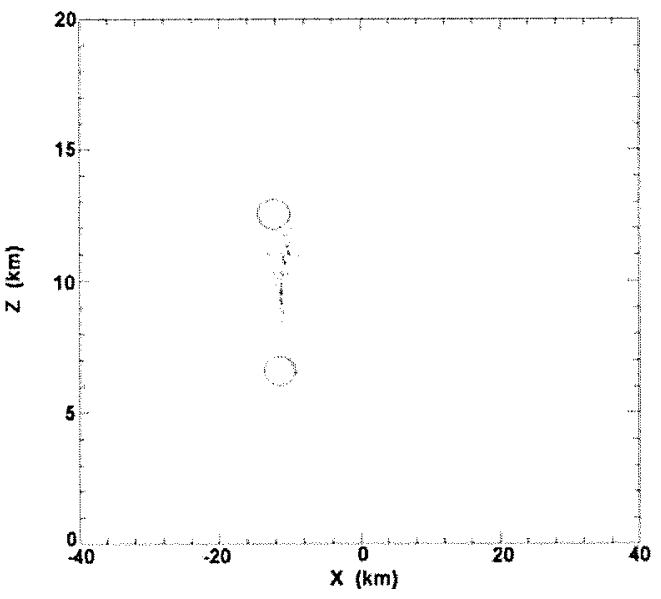

(c)

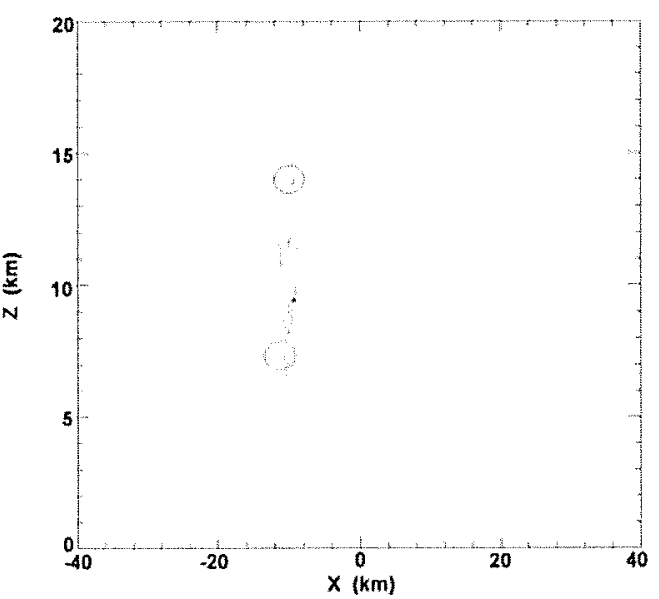

(e)

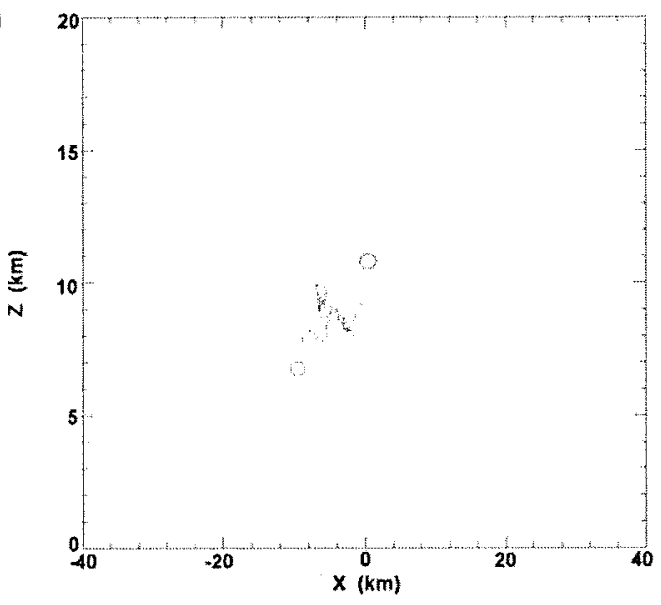

(b)

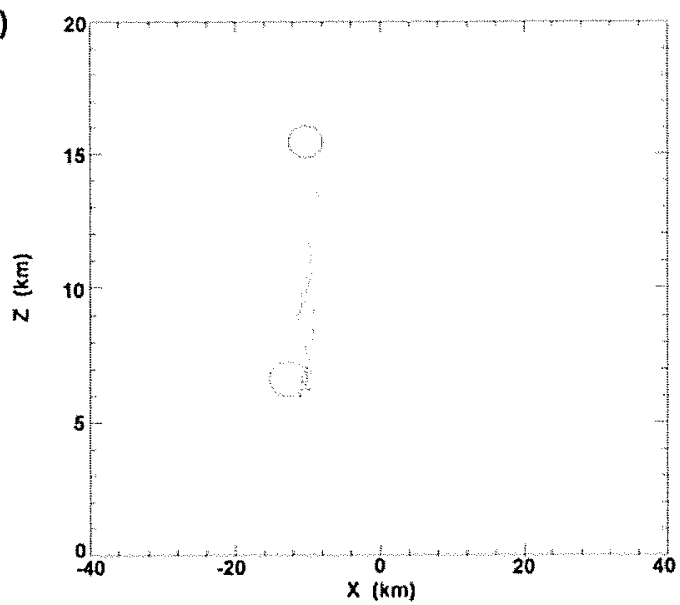

(d)

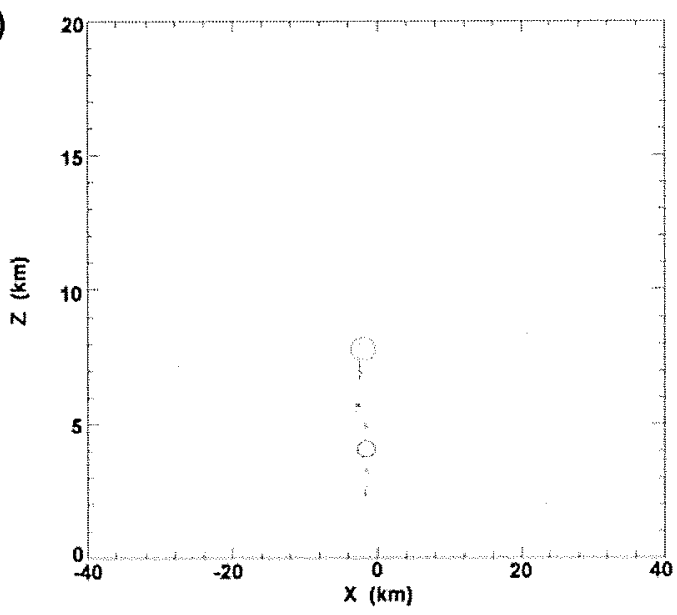

(f)

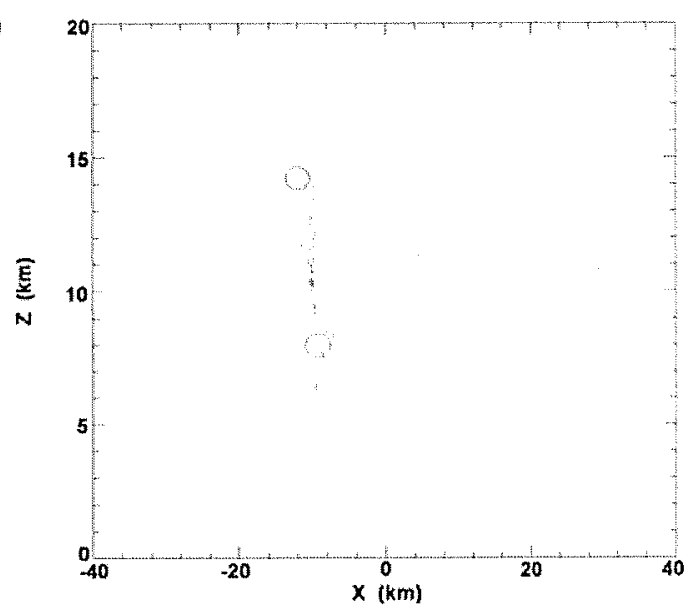

Fig. 6. Examples of charge solutions derived from the DR method (and picked from the results in Figure 5) that compare well with LDAR data. 
(a)

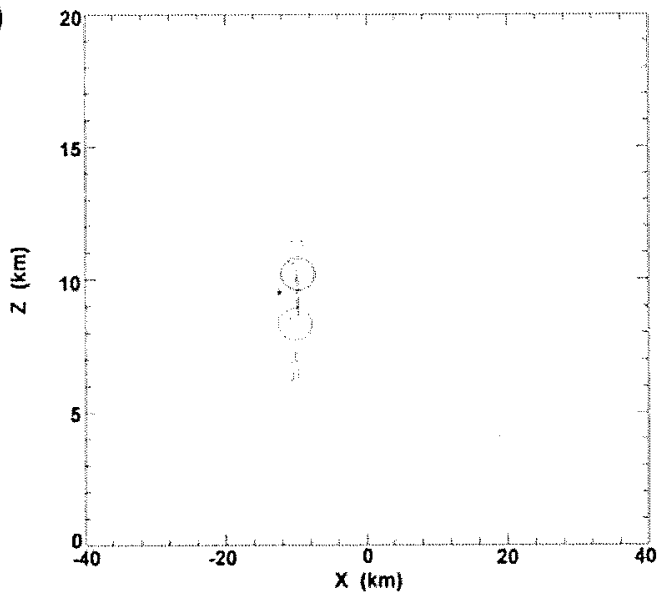

(c)

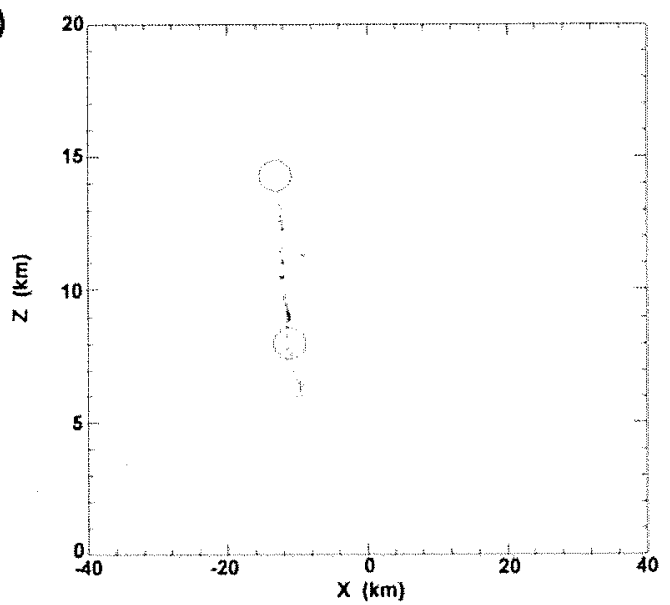

(e)

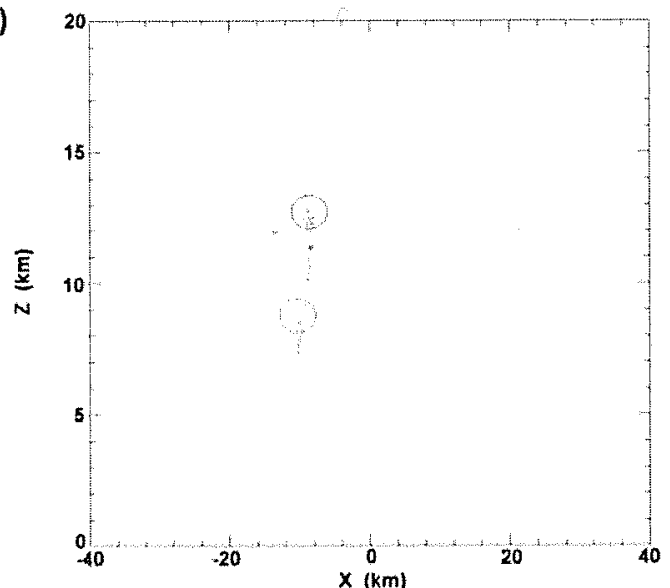

(b)

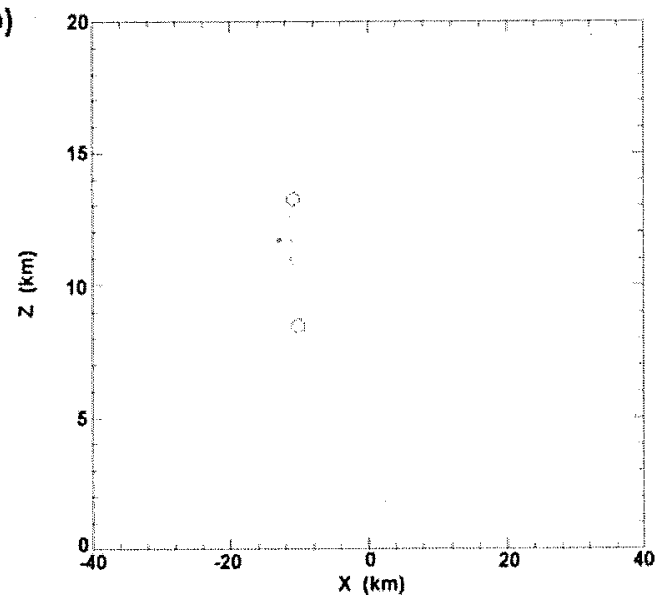

(d)

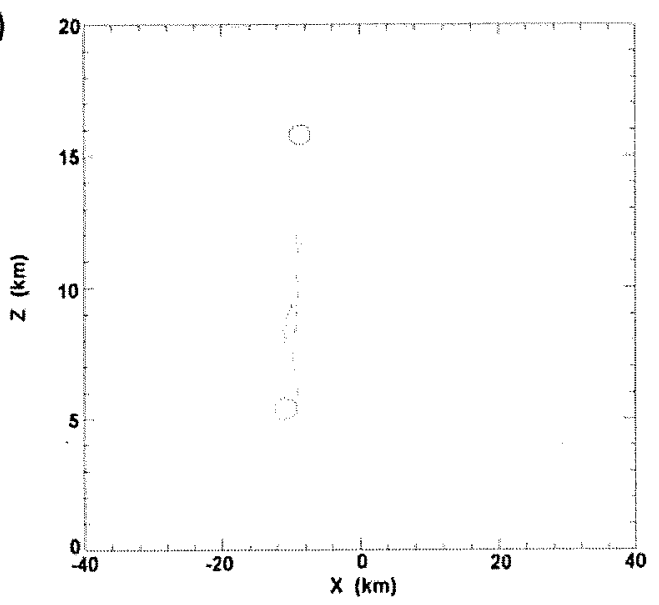

(f)

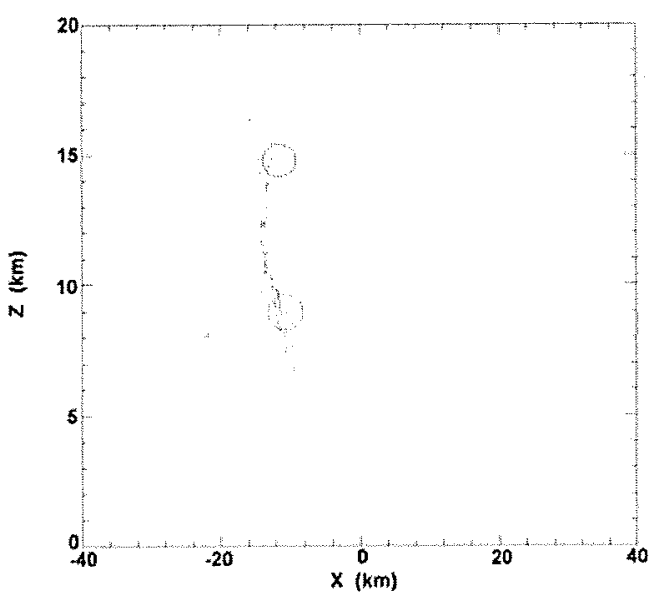

Fig. 7. Examples of charge solutions derived from the DR method (and picked from the results in Figure 5) that show charge location offset errors in the vertical relative to LDAR data. 
(a)

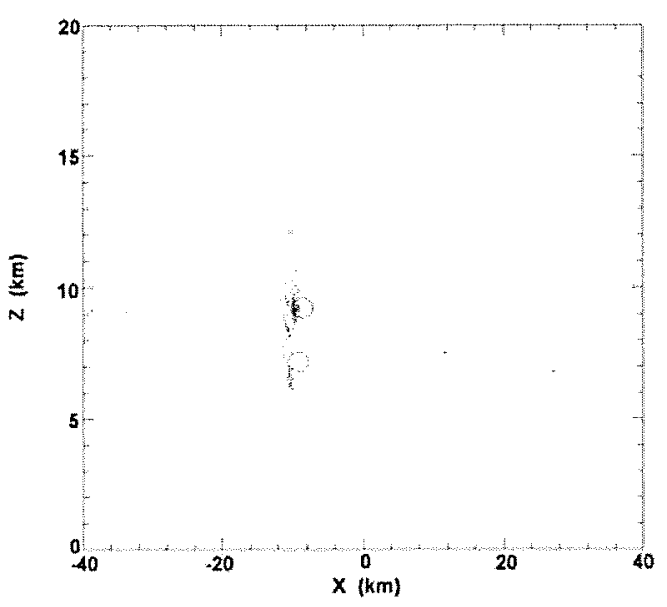

(c)

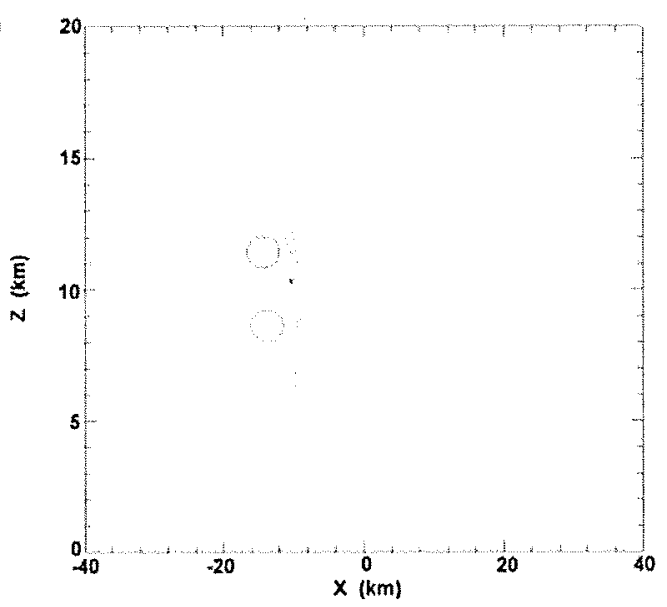

(e)

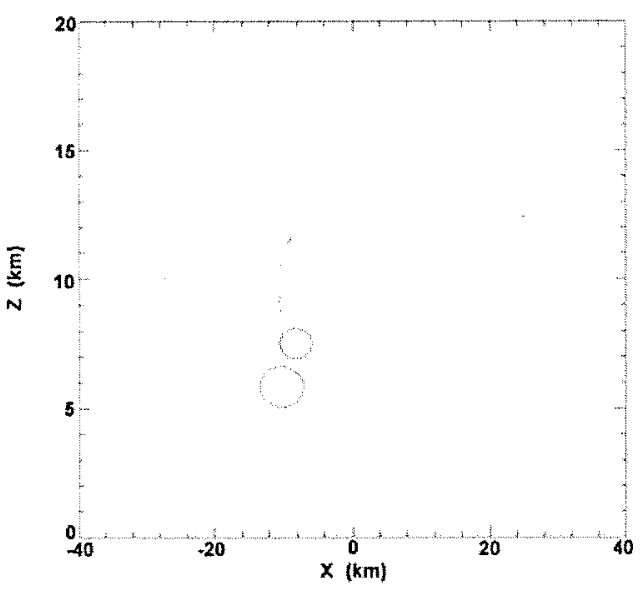

(b)

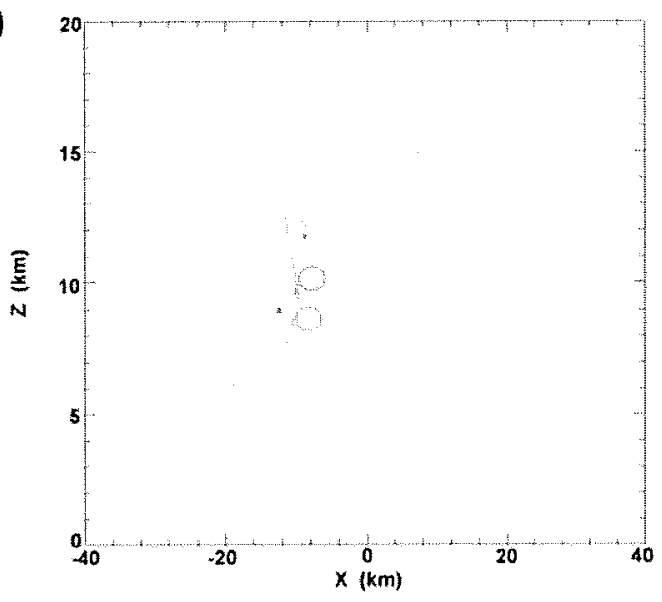

(d)

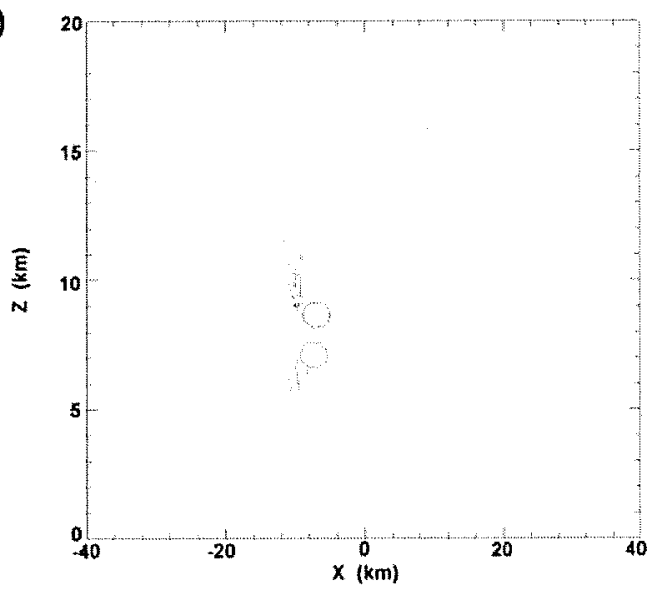

(f)

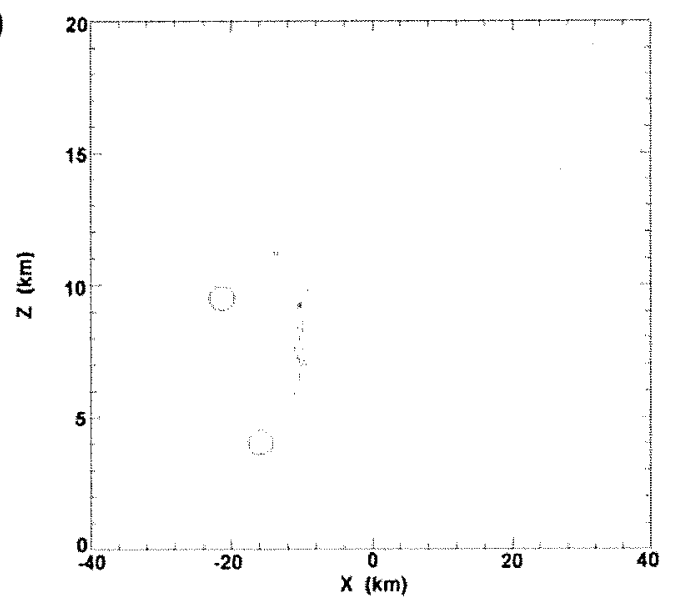

Fig. 8. Examples of flashes derived from the DR method (and picked from the results in Figure 5) that show charge location offset errors in the horizontal and vertical relative to LDAR data. 
(a)

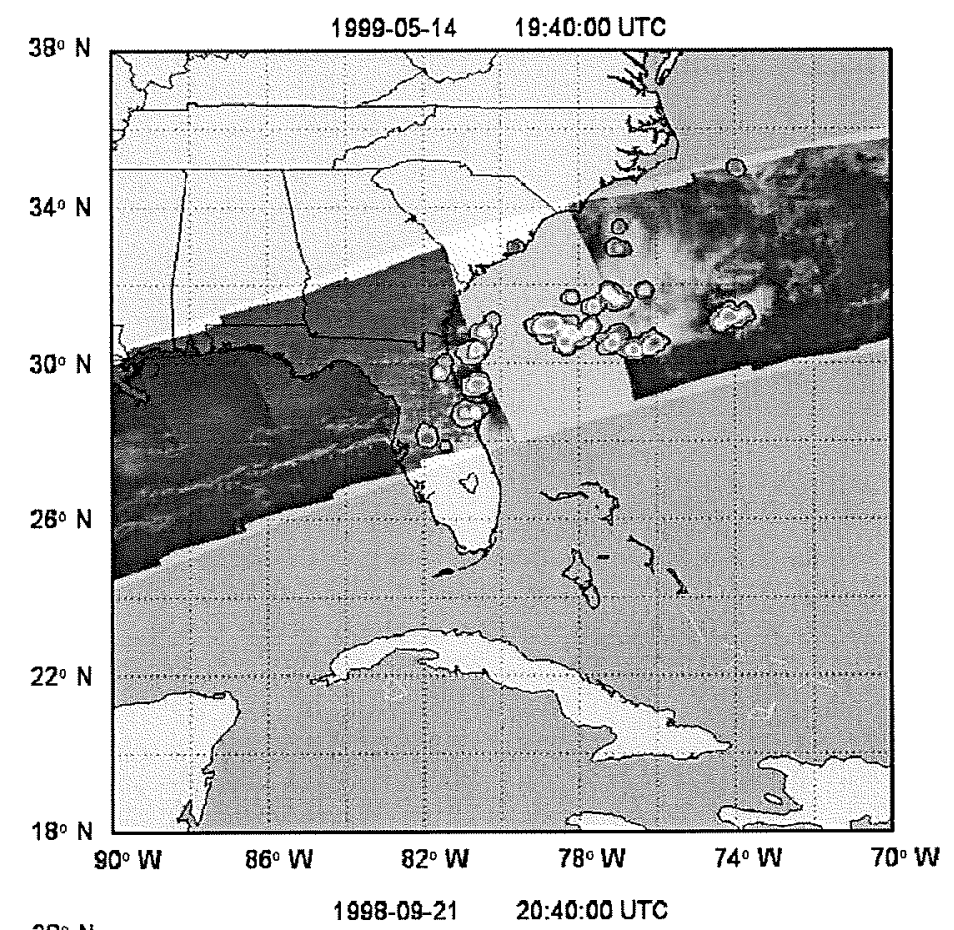

(b)

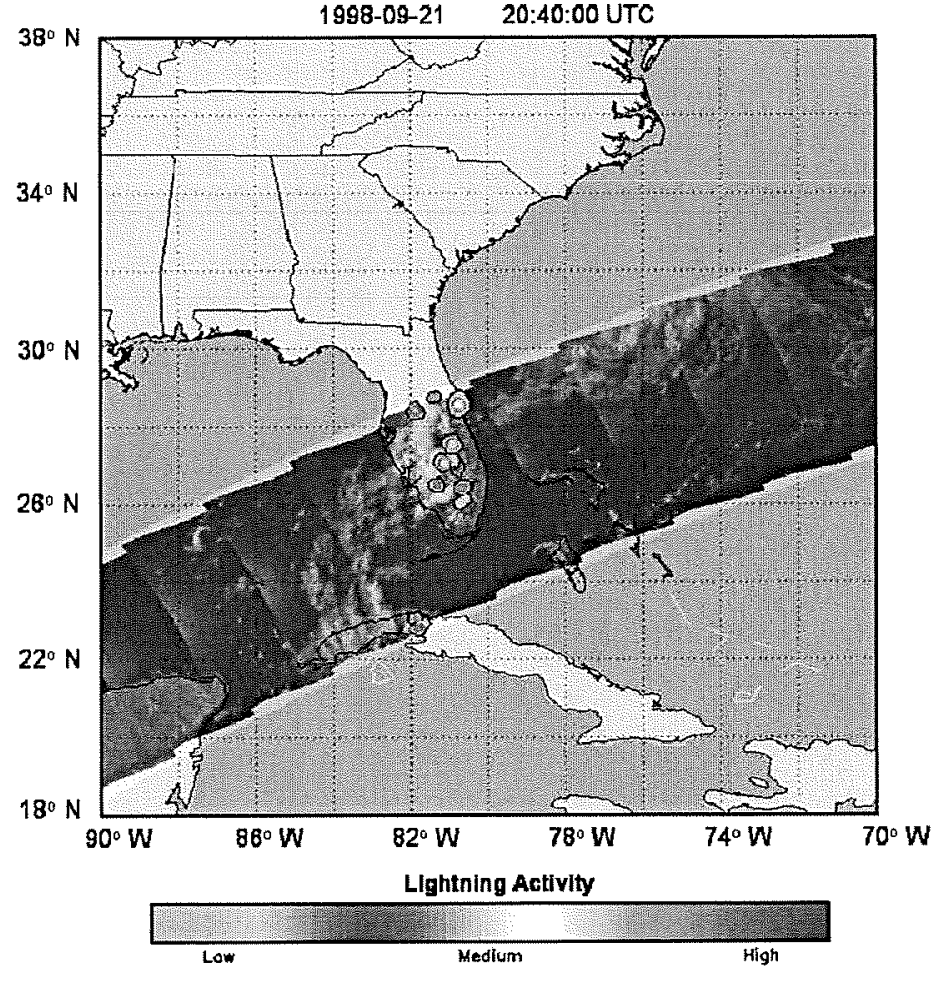

Fig. 9. Examples of two LIS overpasses of KSC on (a) 14 May 1999, and (b) 21 September 1998. The background cloud image is not always collected if lightning rates are high. A total of 7 overpasses (on seven different storm days) were analyzed in this study. 
May 14, 1999 (Day 134)

$\mathrm{Q}=56.168, \mathrm{Q}^{\prime}=-8.209$
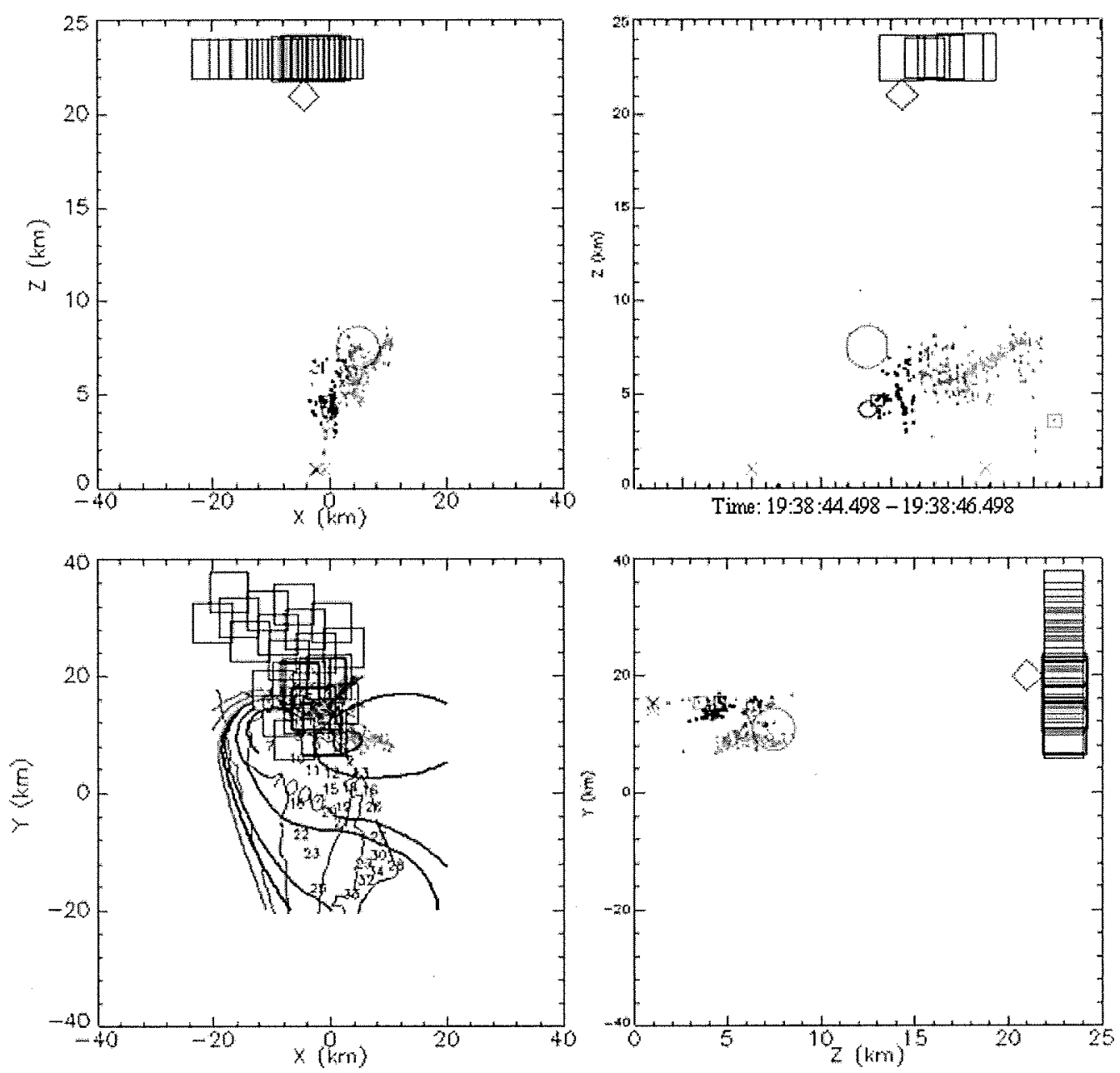

Fig. 10. A CG flash located north of the KSC-ER FM network. The red and blue circles denote lightning-caused changes in thundercloud charge given by $Q$ and $Q^{\prime}$, respectively as derived by using LDAR constraints and linear least squares theory. The values of $Q$ and $Q^{\prime}$ in coulombs are given at the top of the plot. The large squares are individual LIS optical events and the large diamond is the LIS-derived flash location. The small dots indicate VHF source locations for the flash derived from the LDAR network; the small squares identify what VHF source occurred first (black) and last (orange) within the flash. The black and orange "Xs" mark the NLDN- and CGLSS- derived CG location, respectively. 
June 11, 1999 (Day 162)

$\mathrm{Q}=40.072, \mathrm{Q}^{\prime}=-36.123$
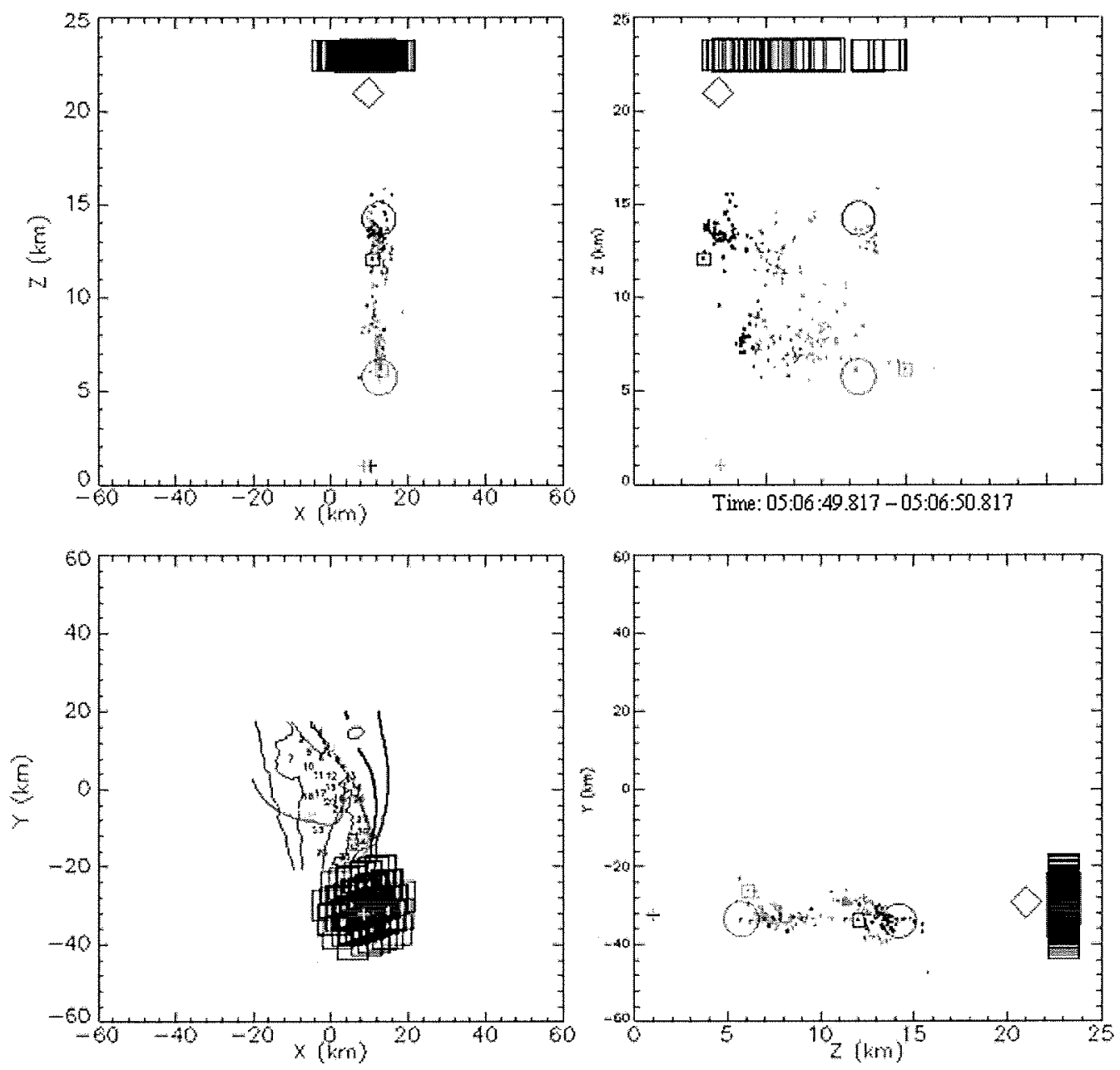

Fig. 11. An IC flash located well south of the KSC-ER network. See caption of Figure 10 for a description of the plot symbols. We believe this flash was incorrectly categorized as a positive CG by the NLDN and CGLSS systems (see main text for a discussion). 

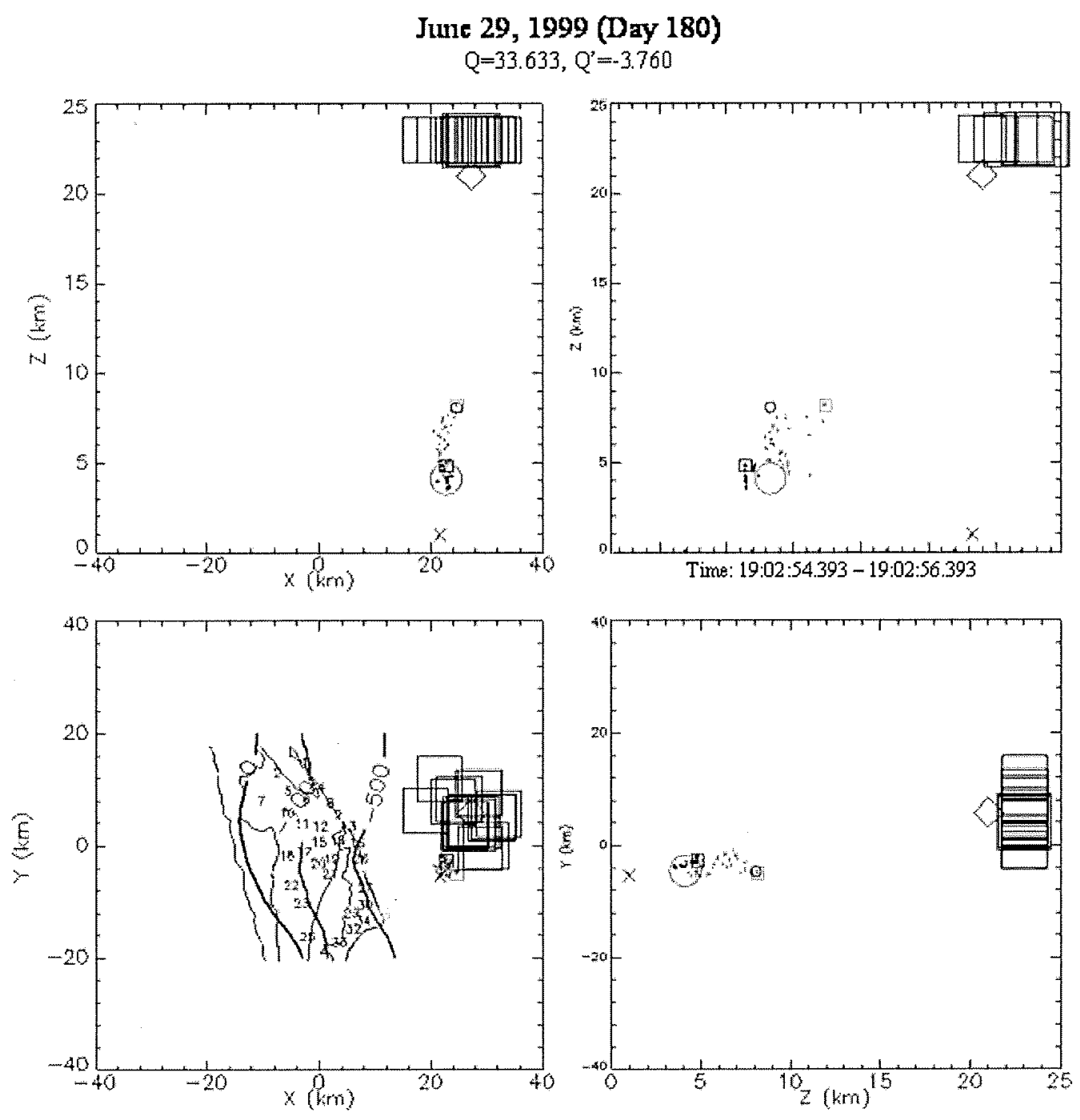

Fig. 12. A CG flash (with IC component) located east of the KSC-ER network. See caption of Figure 10 for a description of the plot symbols. 


\section{September 21, 1998 (Day 264)}

$\mathrm{Q}=9.317, \mathrm{Q}=-3.337$
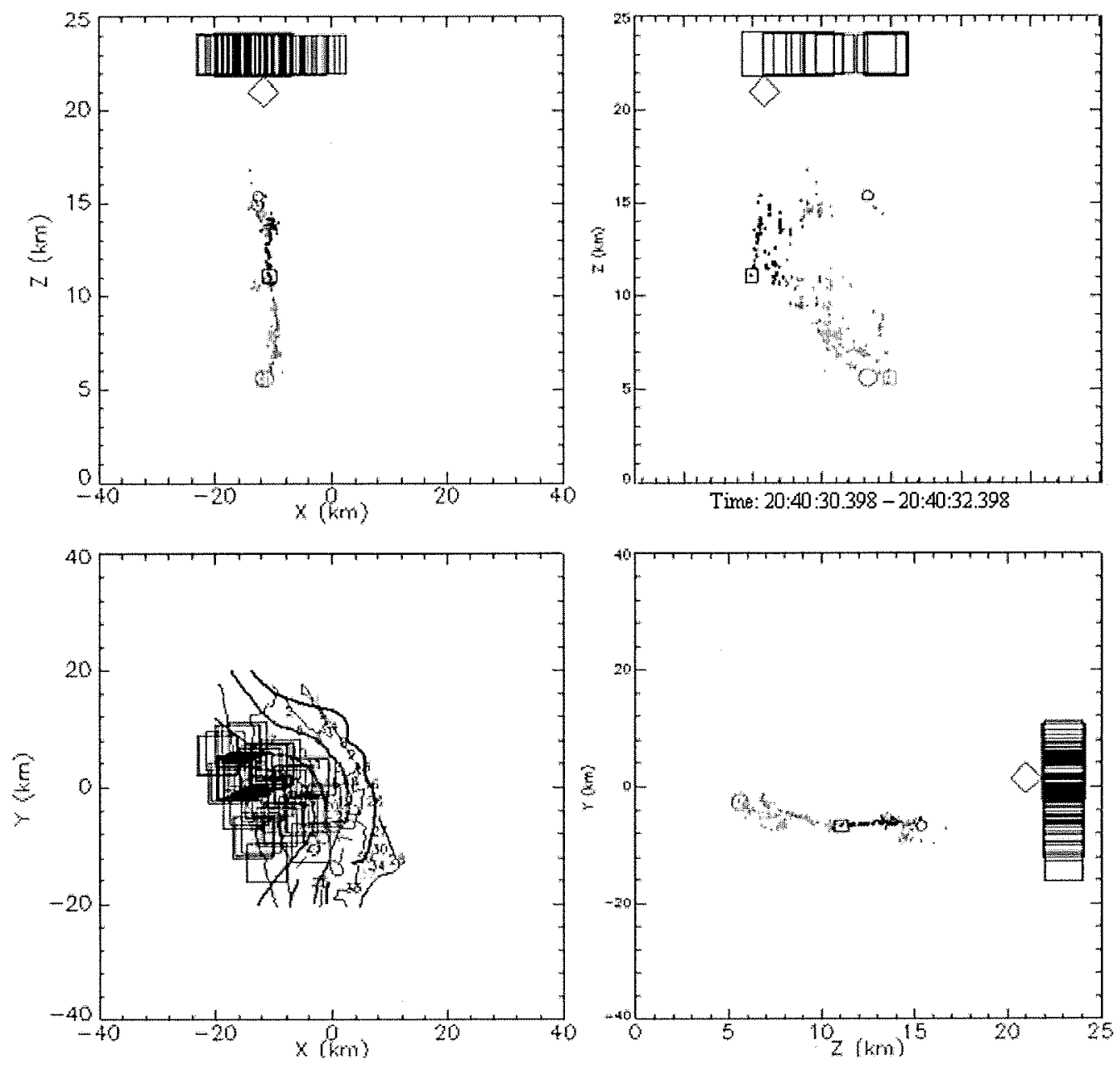

Fig. 13. An IC flash located slightly west of the KSC-ER network. See caption of Figure 10 for a description of the plot symbols. 
September 21, 1998 (Day 264)

$\mathrm{Q}=2.462, \mathrm{Q}^{\prime}=-2.412$
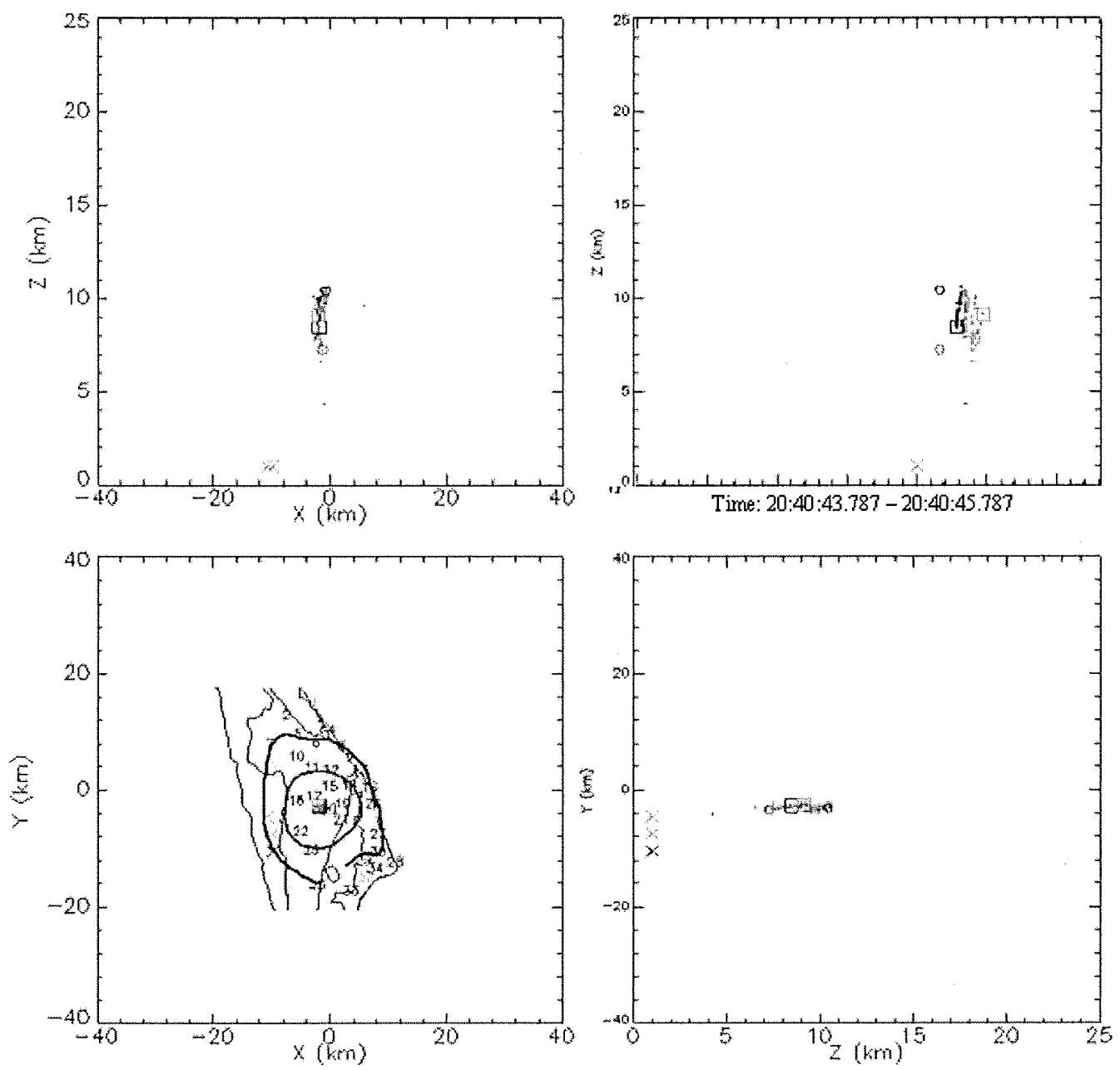

Fig. 14. A small discharge over the KSC-ER network that the LIS instrument detected, but that the LIS data processing algorithm removed. It precedes the CG shown in Figure 15 by less than $0.9 \mathrm{~s}$, and is also within about $10 \mathrm{~km}$ of the CG (see main text for more comments). The CGLSS and NLDN ground strike locations shown are associated with the CG that follows, but appear in this plot due to sensor timing errors. See caption of Figure 10 for a description of the plot symbols. 
September 21, 1998 (Day 264)

$\mathrm{Q}=39.719, \mathrm{Q}^{\prime}=-10.397$
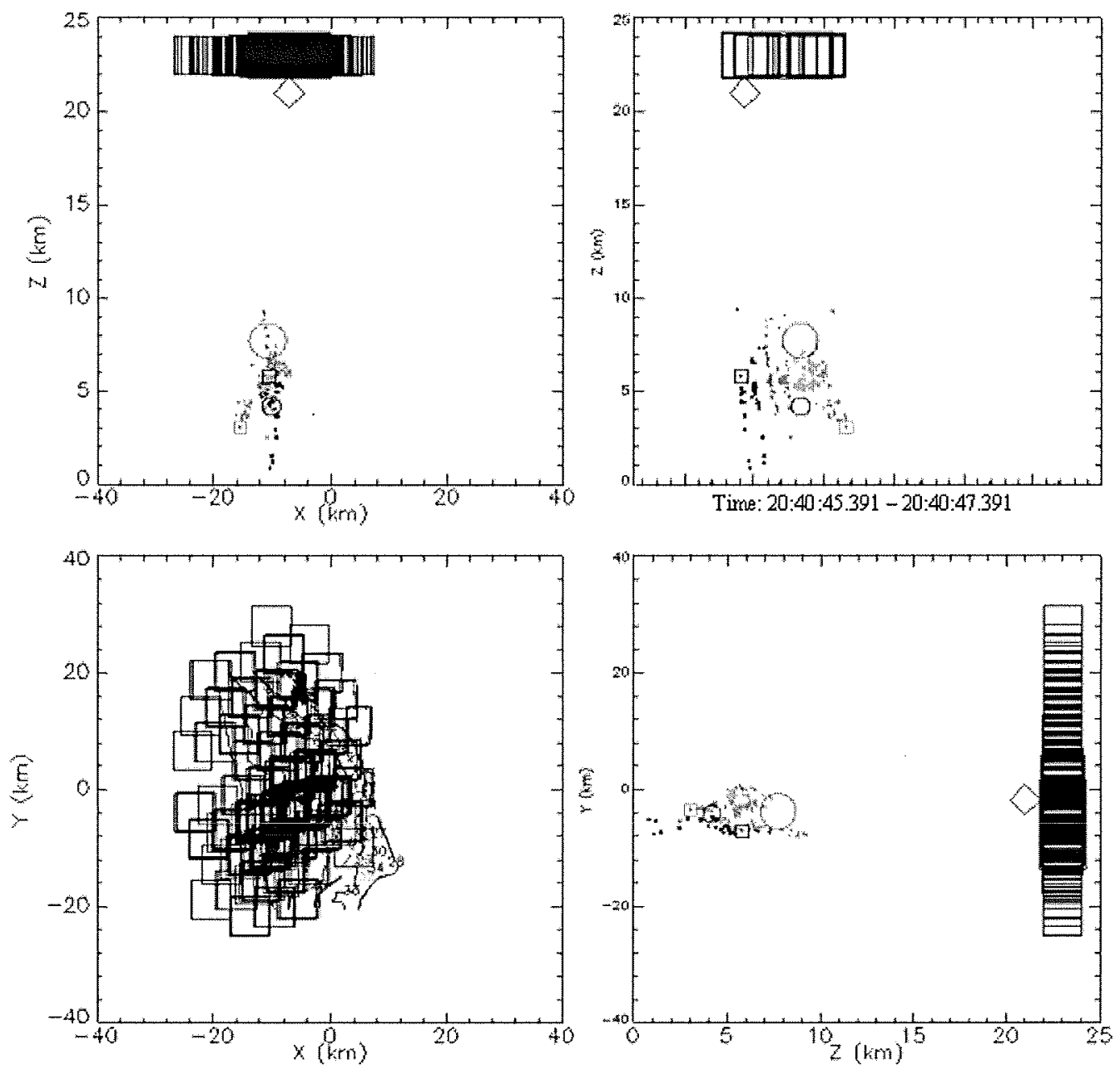

Fig. 15. The CG that quickly follows, and is likely correlated with, the small discharge shown in Figure 14. The CGLSS and NLDN ground strike locations for this CG appear in Figure 14 due to sensor timing errors. See caption of Figure 10 for a description of the plot symbols. 